\title{
Understanding isoprene photooxidation using observations and modeling over a subtropical forest in the southeastern US
}

\section{Luping Su et al.}

Correspondence to: John E. Mak (john.mak@stonybrook.edu )

The copyright of individual parts of the supplement might differ from the CC-BY 3.0 licence. 


\subsection{Peak shape fitting}

3 The peak generated by the PTR-TOF-MS is asymmetric and can be mathematically 4 approximated by peak-shaped functions. Here we choose an empirically transformed 5 Gaussian function (ETG) model to describe a single peak produced by the instrument (Li, 6 1997). The ETG is based on the decomposition of Gaussian function into leading and trailing edge functions. The two functions are weakly combined and subsequently modified, making it very suitable for approximating asymmetric peak shapes. The ETG is given by:

$$
f(t)=\frac{H}{\left\{1+\lambda_{L} \exp \left[k_{L}\left(t_{L}-t\right)\right]\right\}^{\alpha}+\left\{1+\lambda_{T} \exp \left[k_{T}\left(t-t_{T}\right)\right]\right\}^{\beta}-1}
$$

where $\mathrm{f}(\mathrm{t})$ denotes peak function; $\mathrm{t}$ denotes time-of-flight; $\mathrm{H}$ is related to peak height; $\lambda \_\mathrm{L}$ and $\lambda \_T$ are pre-exponential parameters; $k_{\_} L$ and $k_{-} T$ are the parameters related to the speeds of the rise and fall of the leading and trailing edges, respectively; $t \_L$ and $t \_T$ are the inflection times of the leading and trailing edges, respectively, and are fixed values; $\alpha$ and $\beta$ are the parameters to further modify the shapes of the lading and trailing edges, respectively. The ETG features in a weak link between the leading and trailing parts of a single peak, thus is very flexible and suitable for fitting asymmetric peaks. The values of $t \_L$ and $t \_T$ do not need to be accurate, here we use the time-of-flight at the full width at half maximum (FWHM) on the left and right side of a single peak to represent those two values, respectively. There are a total of seven parameters in each ETG $\left(H, \lambda \_L, \lambda \_T, k_{-} L, k_{-} T, \alpha\right.$, and $\left.\beta\right)$, and the rate of convergence is fast. Levenberg-Marquardt algorithm is used to solve the non-linear leastsquares curve fitting problem. To achieve a sufficiently high speed of the curve fitting, $\begin{array}{lllll}\text { MINPACK-1 Least } & \text { Squares Fitting Library in } \mathrm{C}\end{array}$ (http://www.physics.wisc.edu/ craigm/idl/cmpfit.html) is used and integrated into the ToFIND toolbox through MATLAB-C interface (http://www.imm.dtu.dk/ guuru/).

\subsection{Time-of-flight to $\mathrm{m} / \mathrm{z}$ conversion}

In the field free area of the TOF-MS, the time-of-flight of ions show slow drift due to the change in the length of the ions' flight path caused by temperature variations in this area. To gain a high mass accuracy, continuous mass scale correction is performed on the raw data. Four well identified peaks: three internal ions $(\mathrm{H} 218 \mathrm{OH}+, \mathrm{m} / \mathrm{z}=21.022, \mathrm{NO}+, \mathrm{m} / \mathrm{z}=29.997$, 
$4 t=a \sqrt{m / z}+b$

and $\mathrm{H} 216 \mathrm{OH} 218 \mathrm{OH}+, \mathrm{m} / \mathrm{z}=39.033$ ), and one externally added compound (diiodomethane, fragmented at ion $\mathrm{CH} 2 \mathrm{I}+, \mathrm{m} / \mathrm{z}=140.920$ ) are used for mass scale correction peaks. The timeof-flight $t$ and mass scale $\mathrm{m} / \mathrm{z}$ are related to each other through:

For each raw HDF5 data file, every 3 of the 300 spectra are averaged to produce 100 spectra. For each of the 100 spectra, the peak shape fitting algorithm described above is applied to find the peak centers ( $\mathrm{t}$ ) of the four mass scale correction peaks. Since the exact $\mathrm{m} / \mathrm{z}$ values for each peak are known, the values of a and b in Eqn. A2 are calculated through linear leastsquares method. The relative mass error (RME) is less than 10 parts per million (ppm) over the spectrum after mass scale calibration.

\subsection{Peak detection}

After time-of-flight to $\mathrm{m} / \mathrm{z}$ conversion, the 300 mass scale corrected spectra are aligned with each other, and averaged to produce one single spectrum. A target mass list is predefined which includes a series exact $\mathrm{m} / \mathrm{z}$ of interested VOCs compounds (e.g., methanol, acetone, isoprene, MVK+MACR, and monoterpenes). For one specific target peak, there may be other peaks co-existing in the same nominal $\mathrm{m} / \mathrm{z}$, a peak searching routine is used to find all the peaks including the target peak within a certain range of one nominal $\mathrm{m} / \mathrm{z}$. The mass list is then updated and used for peak shape fitting (see next section).

\subsection{Signal integration}

A few issues exist when performing signal integration on each individual peak: (1), due to the low counts rate in each individual $1 \mathrm{~Hz}$ spectrum, the peak shape fitting algorithm often fails to converge or generate desirable fitting results when applied to a single spectrum, (2), interference exists between two neighbouring peaks when they overlap with each other due to the limited mass resolution of the PTR-TOF-MS. To improve the accuracy of the peak fitting result, the updated peak list is used and the peak shape fitting algorithm is applied to the averaged spectrum described above to produce a peak function. Based on this, the FWHM for each peak is recalculated and used to define the peak integration boundaries. For the asymmetrical Gaussian-shaped peak which is typical for the PTR-TOF-MS, the integration boundaries on the left and right sides are processed separately. With the updated FWHM values for all the peaks, the ratios of the portion before the peak center to the portion after the 
1 peak center are calculated and averaged (r). The integration boundary on the left side is

2 defined as $\mathrm{a} \times \mathrm{FWHM} \times \mathrm{r}$ to the left of the peak center. The integration boundary on the right

3 side is defined as a $\times$ FWHM $\times(1-r)$ to the right of the peak center. Add a figure to describe the 4 procedure. The factor a is set to 4 for single peak and 3 for multiple peaks. The factor value is

5 reduced for multiple peaks to lower the interference of neighbouring peaks.

\section{Conversion rate of ISOPOOH to $\mathrm{C}_{4} \mathrm{H}_{7} \mathrm{O}^{+}$in PTR-TOF-MS}

7 To quantify the conversion rate of ISOPOOH to $\mathrm{C}_{4} \mathrm{H}_{7} \mathrm{O}^{+}$when using PTR-TOF-MS, an experiment was carried out by using 1,2-ISOPOOH standard during the Focused Isoprene eXperiment at the California Institute of Technology (FIXCIT) campaign (Nguyen et al.,

21 VOC[molec. $\left.\mathrm{cm}^{-3}\right]=\frac{1}{k t} \times \frac{\mathrm{VOCH}^{+}[\mathrm{cps}]}{\mathrm{H}_{3} \mathrm{O}^{+}[\mathrm{cps}]} \times \sqrt{\frac{\mathrm{m} / \mathrm{z}_{\mathrm{H} 3 \mathrm{O}+}}{\mathrm{m} / \mathrm{z}_{\mathrm{VOCH}+}}} \times \frac{\mathrm{TR}_{\mathrm{H} 3 \mathrm{O}+}}{\mathrm{TR}_{\mathrm{VOCH}+}}$

$22 \quad t=\frac{l^{2}}{\mu_{0} U_{\text {drift }}} \times \frac{T_{0}}{T_{\text {drift }}} \times \frac{p_{\text {drift }}}{p_{0}}$

$\operatorname{air}\left[\right.$ molec. $\left.\mathrm{cm}^{-3}\right]=\frac{N_{A}}{22400} \times \frac{T_{0}}{T_{\text {drift }}} \times \frac{p_{\text {drift }}}{p_{0}}$ the meaning of the symbols are listed as follows, 


\begin{tabular}{|c|c|c|c|}
\hline Symbol & Value & Unit & Description \\
\hline VOC[ppbv] & - & ppbv & VOC mixing ratio \\
\hline VOC $\left[\right.$ molec. $\left.\mathrm{cm}^{-3}\right]$ & - & molec. $\mathrm{cm}^{-3}$ & VOC number density \\
\hline $\operatorname{air}\left[\right.$ molec. $\left.\mathrm{cm}^{-3}\right]$ & - & molec. $\mathrm{cm}^{-3}$ & air number density \\
\hline $\mathrm{VOCH}^{+}[\mathrm{cps}]$ & - & cps & measured $\mathrm{VOCH}^{+}$count rates \\
\hline $\mathrm{H}_{3} \mathrm{O}^{+}[\mathrm{cps}]$ & - & сps & measured primary ion count rates \\
\hline$\mu_{0}$ & 2.8 & $\mathrm{~cm}^{2} \mathrm{~V}^{-1} \mathrm{~s}^{-1}$ & reduced mobility \\
\hline$p_{0}$ & 1013.25 & mbar & air pressure at standard condition \\
\hline$p_{\text {drift }}$ & 2.20 & mbar & drift tube pressure \\
\hline$T_{0}$ & 273.15 & K & temperature at standard condition \\
\hline$T_{\text {drift }}$ & 332.6 & $\mathrm{~K}$ & drift tube temperature \\
\hline$N_{A}$ & $6.022 \times 10^{23}$ & 1 & Avogadro number \\
\hline$l$ & 9.3 & $\mathrm{~cm}$ & length of the reaction chamber \\
\hline$T R_{H 3 O+}$ & 0.67 & 1 & transmission factor of $\mathrm{H}_{3} \mathrm{O}^{+}$ \\
\hline$T R_{\mathrm{VOCH}+}$ & 1.00 & 1 & transmission factor of $\mathrm{VOCH}^{+}$ \\
\hline$k$ & $3.2 \times 10^{-9 a}$ & $\mathrm{~cm}^{3} \mathrm{~s}^{-1}$ & reaction rate coefficient between $\mathrm{VOC}$ and $\mathrm{H}_{3} \mathrm{O}^{+}$ \\
\hline
\end{tabular}

1 Data obtained from Cappellin et al. (2012).

\section{$2 \quad 2.2$ Using sensitivity}

$3 \quad \mathrm{VOC}[\mathrm{ppbv}]=\frac{\mathrm{VOCH}^{+}[\mathrm{ncps}]}{\text { Sensitivity }_{\mathrm{VOCH}+}\left[\mathrm{ncps} \mathrm{ppbv}^{-1}\right]}$

$4 \mathrm{VOCH}^{+}[\mathrm{ncps}]=\frac{\mathrm{VOCH}^{+}[\mathrm{cps}] \times 10^{6}}{\mathrm{H}_{3} \mathrm{O}^{+}[\mathrm{cps}]} \times \sqrt{\frac{\mathrm{m} / \mathrm{z}_{\mathrm{H3O+}}}{\mathrm{m} / \mathrm{z}_{\mathrm{VOCH}+}}}$

5 where Sensitivity $4 \mathrm{CH}_{7+}=26.83 \mathrm{ncps} \mathrm{ppbv}^{-1}$ by using standard gas calibration 
2 The mixing ratios (mean \pm 1 standard deviation) of $\mathrm{m} / \mathrm{z} 71.049$ during experiment step 2 3 (Figure S0) are calculated to be $36.20 \pm 1.62 \mathrm{ppbv}$ and $34.89 \pm 1.54$ ppbv by using reaction 4 rate coefficient and sensitivity, respectively. The difference of mixing ratios between the two 5 methods is within $4 \%$. The mixing ratio of $\mathrm{m} / \mathrm{z} 71.049$ obtained from the sensitivity method is 6 used to calculate the conversion rate of ISOPOOH. The mixing ratio of 1,2-ISOPOOH in the 7 Teflon bag is quantified to be 250 ppbv ( $\pm 40 \%$ uncertainty) (Nguyen et al., 2014) (A. Teng, 8 personal communication). The conversion rate of ISOPOOH to $\mathrm{C}_{4} \mathrm{H}_{7} \mathrm{O}^{+}$is estimated to be $914 \%(+14 \% /-6 \%)$.

10 


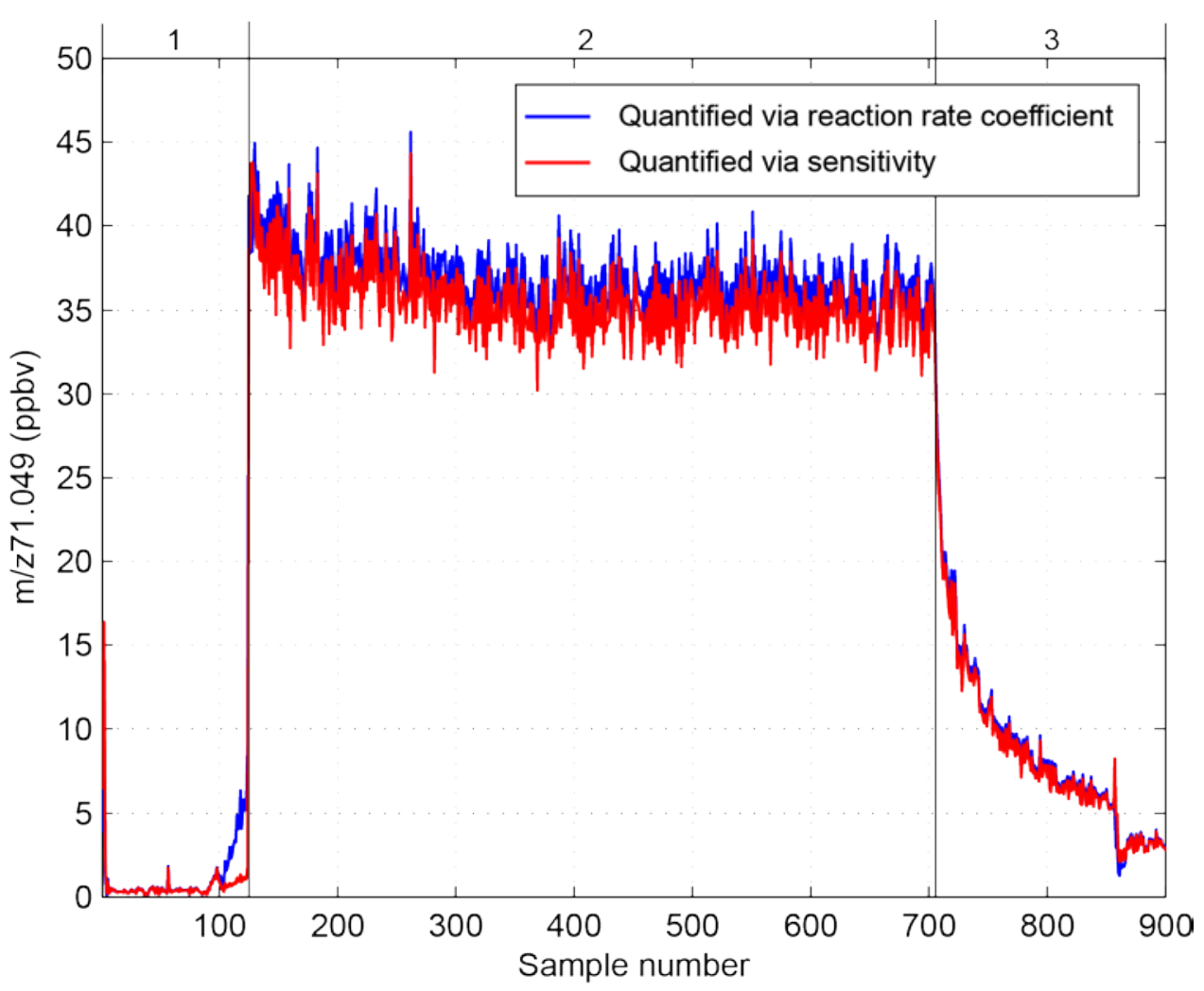

3

4 Figure S0. The mixing ratios of $\mathrm{m} / \mathrm{z} 71.049$ during an ISOPOOH cold trap experiment. 1, 5 sampling from ISOPOOH-free air; 2, sampling from Teflon bag filled with ISOPOOH 6 standard under room temperature through a 1/8 inch OD PFA tubing (length $\sim 40 \mathrm{~cm}$ ) at $\sim 25$ $7 \quad{ }^{\circ} \mathrm{C} ; 3$, same setup as 2 except that the PFA tubing is submerged in cold trap at $-40{ }^{\circ} \mathrm{C}$.

8

9

10

11

12

13

14

15

16 
2 Table S1. Other observations during the 2013 SAS campaign used in this study. Days used

3 are during June 5, 6, 8, 10-13 unless otherwise specified in the table.

\begin{tabular}{|c|c|c|c|c|c|}
\hline Parameter & Platform & Instrument/technique & Uncertainty & Days used & Institute \\
\hline VOC & $\begin{array}{l}\text { Airborne } \\
\text { (NCAR C-130) }\end{array}$ & $\begin{array}{l}\text { Proton Transfer Reaction Mass } \\
\text { Spectrometer (PTR-MS) }\end{array}$ & $20 \%$ & June 12 & $\begin{array}{l}\text { NCAR, } \\
\text { NOAA }\end{array}$ \\
\hline ISOPN & SEARCH site & $\begin{array}{l}\text { chemical ionization mass } \\
\text { spectrometer (CIMS) }\end{array}$ & $25 \%$ & June $5,6,8,10-13$ & $\begin{array}{l}\text { Purdue } \\
\text { University }\end{array}$ \\
\hline O3, NOx & $\begin{array}{l}\text { Airborne } \\
\text { (NCAR C-130) }\end{array}$ & chemiluminescence & $\begin{array}{l}10 \% \text { for } \mathrm{NO}, \\
15 \% \text { for } \\
\mathrm{NO}_{2}, 5 \% \text { for } \\
\mathrm{O}_{3}\end{array}$ & June 12 & NCAR \\
\hline $\mathrm{O} 3, \mathrm{NOx}$ & SEARCH tower & ThermoScientific 49i, $42 \mathrm{i}$ & $\begin{array}{l}\mathrm{NO}, 15.0 \% \\
\text { for } \mathrm{NO}_{2} \text {, } \\
6.1 \% \text { for } \mathrm{O}_{3}\end{array}$ & June 5, 6, 8, 10-13 & ARA \\
\hline $\mathrm{OH}$ & SEARCH tower & laser induced fluorescence (LIF) & $32 \%$ & June 5, 6, 8, 10-13 & $\begin{array}{l}\text { Pennsylvania } \\
\text { State } \\
\text { University }\end{array}$ \\
\hline $\mathrm{HCHO}$ & SEARCH tower & Fiber - Laser Induced Fluoresence & $15 \%$ & June 14 & $\begin{array}{l}\text { University o } \\
\text { Wisconsin- } \\
\text { Madison }\end{array}$ \\
\hline $\begin{array}{l}\text { Surface } \\
\text { heat flux }\end{array}$ & AABC tower & $\begin{array}{l}\text { Integrated Surface Flux System } \\
\text { (ISFS) }\end{array}$ & & June 10-13 & NCAR \\
\hline $\begin{array}{l}\text { Potential } \\
\text { temperature } \\
\text { specific } \\
\text { humidity }\end{array}$ & AABC tower & ISFS & & June 10-13 & NCAR \\
\hline $\begin{array}{l}\text { Potential } \\
\text { temperature } \\
\text { specific } \\
\text { humidity }\end{array}$ & $\begin{array}{l}\text { Airborne } \\
\text { (NCAR C-130) }\end{array}$ & onboard sensor & & June 12 & NCAR \\
\hline $\begin{array}{l}\text { Photolysis } \\
\text { rates }\end{array}$ & $\begin{array}{l}\text { Airborne } \\
\text { (NCAR C-130) }\end{array}$ & onboard sensor & & June 12 & NCAR \\
\hline $\begin{array}{l}\text { Boundary } \\
\text { layer height }\end{array}$ & SEARCH & $\begin{array}{l}\text { Ceilometer, Integrated Sounding } \\
\text { System (ISS) }\end{array}$ & $\begin{array}{l}13.0 \% \text { for } \\
\text { ceilometer }\end{array}$ & June 10-13 & $\begin{array}{l}\text { ARA, } \\
\text { NCAR }\end{array}$ \\
\hline
\end{tabular}

4

5

6 
3 Table S2. Chemical species in the complex scheme. See more details at

4 http:// http://wiki.seas.harvard.edu/geos-chem/index.php/New_isoprene_scheme

\begin{tabular}{|c|c|c|}
\hline Species & Formula & Note \\
\hline A3O2 & СH3CH2CH2OO & primary RO2 from C3H8 \\
\hline ACET & СНЗС (O)СН3 & acetone \\
\hline ACTA & СH3C(O)OH & acetic acid \\
\hline ALD2 & СНЗСНО & acetaldehyde \\
\hline ALK4 & RH & $\geq \mathrm{C} 4$ alkanes \\
\hline ATO2 & $\mathrm{CH} 3 \mathrm{C}(\mathrm{O}) \mathrm{CH} 2 \mathrm{O} 2$ & RO2 from acetone \\
\hline ATOOH & $\mathrm{CH} 3 \mathrm{C}(\mathrm{O}) \mathrm{CH} 2 \mathrm{OOH}$ & ATO2 peroxide \\
\hline $\mathrm{B} 3 \mathrm{O} 2$ & СН3СH(OO)CH3 & secondary RO2 from C3H8 \\
\hline C2H6 & $\mathrm{C} 2 \mathrm{H} 6$ & ethane \\
\hline СЗН8 & С3H8 & propane \\
\hline $\mathrm{CH} 2 \mathrm{O}$ & $\mathrm{CH} 2 \mathrm{O}$ & formaldehyde \\
\hline $\mathrm{CH} 4$ & $\mathrm{CH} 4$ & methane \\
\hline $\mathrm{CO}$ & $\mathrm{CO}$ & carbon monoxide \\
\hline $\mathrm{CO} 2$ & $\mathrm{CO} 2$ & carbon dioxide \\
\hline DHMOB & $\mathrm{HOCH} 2 \mathrm{C}(\mathrm{CH} 3)(\mathrm{OH}) \mathrm{C}(=\mathrm{O}) \mathrm{CHO}$ & See Paulot et al., ACP (2009) \\
\hline DIBOO & & Dibble peroxy radical \\
\hline $\mathrm{EOH}$ & $\mathrm{C} 2 \mathrm{H} 5 \mathrm{OH}$ & ethanol \\
\hline ETHLN & CHOCH2ONO2 & Ethanal nitrate \\
\hline ETO2 & СH3CH2OO & ethylperoxy radical \\
\hline ETP & СН3СН2ОOH & ethylhydroperoxide \\
\hline GLYC & $\mathrm{HOCH} 2 \mathrm{CHO}$ & glycoaldehyde (hydroxyacetaldehyde) \\
\hline GLYX & CHOCHO & glyoxal \\
\hline $\mathrm{H} 2$ & $\mathrm{H} 2$ & hydrogen atom \\
\hline $\mathrm{H} 2 \mathrm{O}$ & $\mathrm{H} 2 \mathrm{O}$ & water vapor \\
\hline $\mathrm{H} 2 \mathrm{O} 2$ & $\mathrm{H} 2 \mathrm{O} 2$ & hydrogen peroxide \\
\hline HAC & $\mathrm{HOCH} 2 \mathrm{C}(\mathrm{O}) \mathrm{CH} 3$ & hydroxyacetone \\
\hline $\mathrm{HCOOH}$ & $\mathrm{HCOOH}$ & formic acid \\
\hline HC5 & $\mathrm{HOCH} 2 \mathrm{CH}=\mathrm{C}(\mathrm{CH} 3) \mathrm{CHO}$ & Hydroxycarbonyl with 5C \\
\hline HC5OO & & Peroxy radical from HC5 (old IAO2?) \\
\hline HNO2 & HONO & nitrous acid \\
\hline HNO3 & HNO3 & nitric acid \\
\hline HNO4 & HNO4 & pernitric acid \\
\hline $\mathrm{HO} 2$ & $\mathrm{HO} 2$ & $\begin{array}{l}\text { hydroperoxyl radical } \\
\text { hydroxy carbonyl alkenes from }\end{array}$ \\
\hline IALD & $\mathrm{HOCH} 2 \mathrm{C}(\mathrm{CH} 3)=\mathrm{CHCHO}$ & isoprene \\
\hline IAP & $\mathrm{HOCH} 2 \mathrm{C}(\mathrm{CH} 3)(\mathrm{OOH}) \mathrm{CH}(\mathrm{OH}) \mathrm{CHO}$ & peroxide from IAO2 \\
\hline IEPOX & & Isoprene epoxide \\
\hline IEPOXOO & & RO2 from IEPOX \\
\hline INO2 & O2NOCH2C(OO)(CH3)CH=CH2 & RO2 from ISOP+NO3 \\
\hline
\end{tabular}




\begin{tabular}{|c|c|c|}
\hline INPN & O2NOCH2C(OOH)(CH3)CH=CH2 & peroxide from INO2 \\
\hline ISN1 & & nighttime isoprene nitrate \\
\hline ISNOOA & & peroxy radical from ISN1 \\
\hline ISNOOB & & peroxy radical from ISN1 \\
\hline ISNOHOO & & $\begin{array}{l}\text { peroxy radical from ISN1 } \\
\text { peroxide from ISOPNBO2 and }\end{array}$ \\
\hline ISNP & $\mathrm{HOCH} 2 \mathrm{C}(\mathrm{OOH})(\mathrm{CH} 3) \mathrm{CH}(\mathrm{ONO} 2) \mathrm{CH} 2 \mathrm{OH}$ & ISOPNDO2 \\
\hline ISOP & $\mathrm{CH} 2=\mathrm{C}(\mathrm{CH} 3) \mathrm{CH}=\mathrm{CH} 2$ & isoprene \\
\hline ISOPNB & C5H9NO4 & Isoprene nitrate Beta \\
\hline ISOPND & C5H9NO4 & Isoprene nitrate Delta \\
\hline KO2 & RO2 from $>3$ ketones & RO2 from $>3$ ketones \\
\hline M & & for three body reactions \\
\hline MACR & $\mathrm{CH} 2=\mathrm{C}(\mathrm{CH} 3) \mathrm{CHO}$ & methacrolein \\
\hline MACRN & HOCH2C(ONO2)(CH3)CHO & Nitrate from MVK \\
\hline MAN2 & $\mathrm{HOCH} 2 \mathrm{C}(\mathrm{ONO} 2)(\mathrm{CH} 3) \mathrm{CHO}$ & $\mathrm{RO} 2$ from MACR+NO3 \\
\hline MAO3 & $\mathrm{CH} 2=\mathrm{C}(\mathrm{CH} 3) \mathrm{C}(\mathrm{O}) \mathrm{OO}$ & peroxyacyl from MVK and MACR \\
\hline MAOP & $\mathrm{CH} 2=\mathrm{C}(\mathrm{CH} 3) \mathrm{C}(\mathrm{O}) \mathrm{OOH}$ & peroxide from MAO3 \\
\hline MAOPO2 & СH2OH-CHOO* $\mathrm{CH} 3 \mathrm{C}(\mathrm{O}) \mathrm{OOH}$ & $\begin{array}{l}\text { Peroxy radical from MAOP (addition } \\
\text { on the double bond) }\end{array}$ \\
\hline MAP & СН3С(O)OOH & peroxyacetic acid \\
\hline MCO3 & СН3С $(\mathrm{O}) \mathrm{OO}$ & peroxyacetyl radical \\
\hline MEK & $\mathrm{RC}(\mathrm{O}) \mathrm{R}$ & $>3$ ketones \\
\hline MGLY & СНЗСОСНО & methylglyoxyal \\
\hline MNO3 & CH3ONO2 & methylnitrate \\
\hline MOBA & $\mathrm{HOC}(=\mathrm{O}) \mathrm{C}(\mathrm{CH} 3)=\mathrm{CHCHO}$ & 5C acid from isoprene \\
\hline MOBAOO & & $\mathrm{RO} 2$ from MOBA \\
\hline MO2 & CH3O2 & methylperoxy radical \\
\hline $\mathrm{MOH}$ & СНЗОН & methanol \\
\hline MP & СНЗООН & methylhydroperoxide \\
\hline MRO2 & HOCH2C(OO)(CH3)CHO & $\mathrm{RO} 2$ from MACR+OH \\
\hline MRP & HOCH2C(OOH)(CH3)CHO & peroxide from MRO2 \\
\hline MVK & $\mathrm{CH} 2=\mathrm{CHC}(=\mathrm{O}) \mathrm{CH} 3$ & methylvinylketone \\
\hline MVKN & $\mathrm{HOCH} 2 \mathrm{CH}(\mathrm{ONO} 2) \mathrm{C}(=\mathrm{O}) \mathrm{CH} 3$ & Nitrate from MACR \\
\hline N2 & N2 & nitrogen \\
\hline $\mathrm{N} 2 \mathrm{O}$ & $\mathrm{N} 2 \mathrm{O}$ & nitrous oxide \\
\hline N2O5 & N2O5 & dinitrogen pentoxide \\
\hline NH2 & NH2 & ammonia radical \\
\hline NH3 & NH3 & ammonia \\
\hline NO & NO & nitric oxide \\
\hline NO2 & NO2 & nitrogen dioxide \\
\hline NO3 & NO3 & nitrate radical \\
\hline $\mathrm{O} 2$ & $\mathrm{O} 2$ & molecular oxygen \\
\hline $\mathrm{O} 2 \mathrm{CH} 2 \mathrm{OH}$ & $\mathrm{O} 2 \mathrm{CH} 2 \mathrm{OH}$ & produced by $\mathrm{CH} 2 \mathrm{O}+\mathrm{HO} 2$ \\
\hline $\mathrm{O} 3$ & O3 & ozone \\
\hline $\mathrm{OH}$ & $\mathrm{OH}$ & hydroxyl radical \\
\hline PAN & СН3C(O)OONO2 & peroxyacetylnitrate \\
\hline PMN & $\mathrm{CH} 2=\mathrm{C}(\mathrm{CH} 3) \mathrm{C}(\mathrm{O}) \mathrm{OONO} 2$ & peroxymethacryloyl nitrate (MPAN) \\
\hline
\end{tabular}




\begin{tabular}{|c|c|c|}
\hline $\mathrm{PO} 2$ & $\mathrm{HOCH} 2 \mathrm{CH}(\mathrm{OO}) \mathrm{CH} 3$ & RO2 from isoprene \\
\hline PP & HOCH2CH$(\mathrm{OOH}) \mathrm{CH} 3$ & peroxide from PO2 \\
\hline PPN & СH3СH2C(O)OONO2 & peroxypropionylnitrate \\
\hline PRN1 & O2NOCH2CH(OO)CH3 & $\mathrm{RO} 2$ from propene $+\mathrm{NO} 3$ \\
\hline PRPE & C3H6 & $\geq \mathrm{C} 4$ alkenes \\
\hline PRPN & O2NOCH2CH(OOH)CH3 & peroxide from PRN1 \\
\hline PROPNN & CH3C $(=\mathrm{O}) \mathrm{CH} 2 \mathrm{ONO} 2$ & Propanone nitrate \\
\hline PYAC & СНЗСОСООН & Pyruvic acid \\
\hline R4N1 & RO2 from R4N2 & $\mathrm{RO} 2$ from R4N2 \\
\hline R4N2 & RO2NO & $\geq \mathrm{C} 4$ alkylnitrates \\
\hline $\mathrm{R} 4 \mathrm{O} 2$ & RO2 from ALK4 & RO2 from ALK4 \\
\hline R4P & СH3CH2CH2CH2OOH & peroxide from $\mathrm{R} 4 \mathrm{O} 2$ \\
\hline RA3P & СН3СН2CH2OOH & peroxide from $\mathrm{A} 3 \mathrm{O} 2$ \\
\hline RB3P & СНЗСН(OOH)CH3 & peroxide from $\mathrm{B} 3 \mathrm{O} 2$ \\
\hline RCHO & СН3СН2CHO & >C2 aldehydes \\
\hline RCO3 & СН3СH2C(O)OO & peroxypropionyl radical \\
\hline $\mathrm{RCOOH}$ & $\mathrm{C} 2 \mathrm{H} 5 \mathrm{C}(\mathrm{O}) \mathrm{OH}$ & $>\mathrm{C} 2$ organic acids \\
\hline RIO1 & $\mathrm{HOCH} 2 \mathrm{C}(\mathrm{OO})(\mathrm{CH} 3) \mathrm{CH}=\mathrm{CHOH}$ & RO2 from isoprene oxidation products \\
\hline RIO2 & $\mathrm{HOCH} 2 \mathrm{C}(\mathrm{OO})(\mathrm{CH} 3) \mathrm{CH}=\mathrm{CH} 2$ & $\begin{array}{l}\text { RO2 from isoprene (named as ISOPO2 } \\
\text { in the literature) } \\
\text { peroxide from RIO2 (named as }\end{array}$ \\
\hline RIP & $\mathrm{HOCH} 2 \mathrm{C}(\mathrm{OOH})(\mathrm{CH} 3) \mathrm{CH}=\mathrm{CH} 2$ & ISOPOOH in the literature) \\
\hline $\mathrm{ROH}$ & С3H7OH & $>\mathrm{C} 2$ alcohols \\
\hline $\mathrm{RP}$ & СН3СН2C(O)OOH & peroxide from RCO3 \\
\hline VRO2 & $\mathrm{HOCH} 2 \mathrm{CH}(\mathrm{OO}) \mathrm{C}(\mathrm{O}) \mathrm{CH} 3$ & $\mathrm{RO} 2$ from $\mathrm{MVK}+\mathrm{OH}$ \\
\hline VRP & $\mathrm{HOCH} 2 \mathrm{CH}(\mathrm{OOH}) \mathrm{C}(\mathrm{O}) \mathrm{CH} 3$ & peroxide from VRO2 \\
\hline
\end{tabular}


3 Table S3. The complex chemical scheme used in MXLCH.

\begin{tabular}{|c|c|}
\hline Reaction & Reaction rate \\
\hline $\mathrm{O}+\mathrm{O} 2+\mathrm{M}->\mathrm{O} 3+\mathrm{M}$ & $6 \mathrm{E}-34 *(300 / \mathrm{T})^{\wedge} 2.4$ \\
\hline $\mathrm{O}+\mathrm{O} 3->2 \mathrm{O} 2$ & $8.00 \mathrm{E}-12 * \exp (-2060 / \mathrm{T})$ \\
\hline $\mathrm{O} 1 \mathrm{D}+\mathrm{N} 2->\mathrm{O}+\mathrm{N} 2$ & $2.10 \mathrm{E}-11 * \exp (115 / \mathrm{T})$ \\
\hline $\mathrm{O} 1 \mathrm{D}+\mathrm{O} 2->\mathrm{O}+\mathrm{O} 2$ & 3.20E-11*exp(70/T) \\
\hline $\mathrm{O} 1 \mathrm{D}+\mathrm{H} 2 \mathrm{O}->2 \mathrm{OH}$ & 2.20E-10 \\
\hline $\mathrm{H} 2+\mathrm{O} 1 \mathrm{D}->\mathrm{HO} 2+\mathrm{OH}$ & $1.10 \mathrm{E}-10$ \\
\hline $\mathrm{H} 2+\mathrm{OH}->\mathrm{H} 2 \mathrm{O}+\mathrm{HO} 2$ & $5.50 \mathrm{E}-12 * \exp (-2000 / \mathrm{T})$ \\
\hline $\mathrm{O}+\mathrm{OH}->\mathrm{HO} 2+\mathrm{O} 2$ & $2.20 \mathrm{E}-11 * \exp (120 / \mathrm{T})$ \\
\hline $\mathrm{HO} 2+\mathrm{O}->\mathrm{OH}+\mathrm{O} 2$ & 3.00E-11*exp $(200 / \mathrm{T})$ \\
\hline $\mathrm{OH}+\mathrm{O} 3->\mathrm{HO} 2+\mathrm{O} 2$ & $1.70 \mathrm{E}-12 * \exp (-940 / \mathrm{T})$ \\
\hline $\mathrm{HO} 2+\mathrm{O} 3->\mathrm{OH}+2 \mathrm{O} 2$ & $\begin{array}{l}1.00 \mathrm{E}-14 * \exp (-490 / \mathrm{T}) \\
\left(2.3 \mathrm{E}-13^{*} \exp (600 / \mathrm{T})+1.7 \mathrm{E}-\right. \\
\left.33 *[\mathrm{M}]^{*} \exp (1000 / \mathrm{T})\right)^{*}(1+ \\
1.4 \mathrm{E}-\end{array}$ \\
\hline $2 \mathrm{HO} 2->\mathrm{H} 2 \mathrm{O} 2+\mathrm{O} 2$ & $21 *[\mathrm{H} 2 \mathrm{O}] * \exp (2200 / \mathrm{T}))$ \\
\hline $\mathrm{H} 2 \mathrm{O} 2+\mathrm{OH}$-> H2O + HO2 & $2.90 \mathrm{E}-12 * \exp (-160 / \mathrm{T})$ \\
\hline $\mathrm{OH}+\mathrm{HO} 2->\mathrm{H} 2 \mathrm{O}+\mathrm{O} 2$ & $4.80 \mathrm{E}-11 * \exp (250 / \mathrm{T})$ \\
\hline $2 \mathrm{OH}->\mathrm{H} 2 \mathrm{O}+\mathrm{O}$ & $4.20 \mathrm{E}-12 * \exp (-240 / \mathrm{T})$ \\
\hline $2 \mathrm{OH}$-> H2O2 & $6.29 \mathrm{E}-12$ \\
\hline $\mathrm{N} 2 \mathrm{O}+\mathrm{O} 1 \mathrm{D}->\mathrm{N} 2+\mathrm{O} 2$ & $4.90 \mathrm{E}-11$ \\
\hline $\mathrm{N} 2 \mathrm{O}+\mathrm{O} 1 \mathrm{D}->2 \mathrm{NO}$ & $6.70 \mathrm{E}-11$ \\
\hline $\mathrm{NO}+\mathrm{HO} 2->\mathrm{NO} 2+\mathrm{OH}$ & $3.50 \mathrm{E}-12 * \exp (250 / \mathrm{T})$ \\
\hline $\mathrm{NO}+\mathrm{O} 3$-> NO2 + O2 & $3.00 \mathrm{E}-12 * \exp (-1500.0 / \mathrm{T})$ \\
\hline $\mathrm{NO} 2+\mathrm{O}->\mathrm{NO}+\mathrm{O} 2$ & $5.60 \mathrm{E}-12 * \exp (180 / \mathrm{T})$ \\
\hline $\mathrm{NO} 2+\mathrm{O} 3->\mathrm{NO} 3+\mathrm{O} 2$ & $1.20 \mathrm{E}-13 * \exp (-2450 / \mathrm{T})$ \\
\hline $\mathrm{NO} 3+\mathrm{HO} 2->\mathrm{OH}+\mathrm{NO} 2$ & 2.30E-12*exp $(170 / \mathrm{T})$ \\
\hline $\mathrm{NO} 2+\mathrm{NO} 3$-> N2O5 & $1.16 \mathrm{E}-12$ \\
\hline $\mathrm{N} 2 \mathrm{O} 5$-> NO2 + NO3 & 4.70E-02 \\
\hline $\mathrm{NO} 2+\mathrm{OH}->\mathrm{HNO} 3$ & $3.50 \mathrm{E}-12 * \exp (340 / \mathrm{T})$ \\
\hline $\mathrm{HNO}+\mathrm{OH}->\mathrm{NO} 3$ & $1.59 \mathrm{E}-13$ \\
\hline $\mathrm{NO} 3+\mathrm{NO}->2 \mathrm{NO} 2$ & $1.50 \mathrm{E}-11 * \exp (170 / \mathrm{T})$ \\
\hline $\mathrm{NO} 2+\mathrm{HO} 2$-> HO2NO2 & $1.37 \mathrm{E}-12$ \\
\hline $\mathrm{HO} 2 \mathrm{NO} 2+\mathrm{OH}->\mathrm{H} 2 \mathrm{O}+\mathrm{NO} 2+\mathrm{O} 2$ & $1.30 \mathrm{E}-12 * \exp (380 / \mathrm{T})$ \\
\hline $\mathrm{HO} 2 \mathrm{NO} 2$-> HO2 + NO2 & 8.50E-02 \\
\hline $\mathrm{CH} 4+\mathrm{OH}->\mathrm{MO} 2+\mathrm{H} 2 \mathrm{O}$ & $2.45 \mathrm{E}-12 * \exp (-1775 / \mathrm{T})$ \\
\hline $\mathrm{CH} 4+\mathrm{O} 1 \mathrm{D}->.75 \mathrm{MO} 2+.75 \mathrm{OH}+.25 \mathrm{CH} 2 \mathrm{O}+.4 \mathrm{HO} 2+.05 \mathrm{H} 2$ & $1.50 \mathrm{E}-10$ \\
\hline $\mathrm{MO} 2+\mathrm{NO}->\mathrm{CH} 2 \mathrm{O}+\mathrm{NO} 2+\mathrm{HO} 2$ & $2.80 \mathrm{E}-12 * \exp (300 / \mathrm{T})$ \\
\hline $2 \mathrm{MO} 2$-> 2CH2O + 2HO2 & $5.00 \mathrm{E}-13 * \exp (-424 / \mathrm{T})$ \\
\hline $2 \mathrm{MO} 2->\mathrm{CH} 2 \mathrm{O}+\mathrm{MOH}$ & $1.90 \mathrm{E}-14 * \exp (706 / \mathrm{T})$ \\
\hline $\mathrm{MO} 2+\mathrm{HO} 2->\mathrm{MP}+\mathrm{O} 2$ & 4.10E-13*exp(750/T) \\
\hline $\mathrm{MP}+\mathrm{OH}->.7 \mathrm{MO} 2+.3 \mathrm{OH}+.3 \mathrm{CH} 2 \mathrm{O}+\mathrm{H} 2 \mathrm{O}$ & $3.80 \mathrm{E}-12 * \exp (200 / \mathrm{T})$ \\
\hline
\end{tabular}




\begin{tabular}{|c|c|}
\hline $\mathrm{CH} 2 \mathrm{O}+\mathrm{OH}->\mathrm{CO}+\mathrm{H} 2 \mathrm{O}+\mathrm{HO} 2$ & $9.00 \mathrm{E}-12$ \\
\hline $\mathrm{CO}+\mathrm{OH}->(\mathrm{CO} 2)+\mathrm{HO} 2$ & $2.40 \mathrm{E}-13$ \\
\hline $\mathrm{MOH}+\mathrm{OH}->\mathrm{HO} 2+\mathrm{CH} 2 \mathrm{O}$ & 7.30E-12*exp(-620/T) \\
\hline ISOP + OH -> RIO2 & $3.1 \mathrm{E}-11 * \exp (350 / \mathrm{T})$ \\
\hline $\mathrm{MACR}+\mathrm{OH}->0.53 \mathrm{MAO} 3+0.47 \mathrm{MRO} 2$ & $8.0 \mathrm{E}-12 * \exp (380 / \mathrm{T})$ \\
\hline $\mathrm{MVK}+\mathrm{OH}->\mathrm{VRO} 2$ & $2.6 \mathrm{E}-12 * \exp (610 / \mathrm{T})$ \\
\hline $\mathrm{PMN}+\mathrm{OH}->\mathrm{HAC}+\mathrm{CO}+\mathrm{NO} 2$ & $2.90 \mathrm{E}-11$ \\
\hline $\begin{array}{l}\text { GLYC + OH -> 0.732CH2O + }(0.361 \mathrm{CO} 2)+0.505 \mathrm{CO}+0.227 \mathrm{OH}+ \\
0.773 \mathrm{HO} 2+0.134 \mathrm{GLYX}+0.134 \mathrm{HCOOH}\end{array}$ & $6.54 \mathrm{E}-12$ \\
\hline $\mathrm{GLYC}+\mathrm{OH}->\mathrm{HCOOH}+\mathrm{OH}+\mathrm{CO}$ & $1.45 \mathrm{E}-12$ \\
\hline $\mathrm{GLYX}+\mathrm{OH}->\mathrm{HO} 2+2 \mathrm{CO}$ & $3.1 \mathrm{E}-12 * \exp (340 / \mathrm{T})$ \\
\hline $\mathrm{HAC}+\mathrm{OH}->\mathrm{MGLY}+\mathrm{HO} 2$ & $1.81 \mathrm{E}-12 * \exp (305 / \mathrm{T})$ \\
\hline $\begin{array}{l}\mathrm{HAC}+\mathrm{OH}->0.5 \mathrm{HCOOH}+\mathrm{OH}+0.5 \mathrm{ACTA}+(0.5 \mathrm{CO} 2)+0.5 \mathrm{CO}+ \\
0.5 \mathrm{MO} 2\end{array}$ & $3.43 \mathrm{E}-13 * \exp (305 / \mathrm{T})$ \\
\hline $\mathrm{PRPN}+\mathrm{OH}->0.209 \mathrm{PRN} 1+0.791 \mathrm{OH}+0.791 \mathrm{PROPNN}$ & $8.78 \mathrm{E}-12 * \exp (200 / \mathrm{T})$ \\
\hline $\mathrm{ETP}+\mathrm{OH}->0.64 \mathrm{OH}+0.36 \mathrm{ETO} 2+0.60 \mathrm{ALD} 2$ & $5.18 \mathrm{E}-12 * \exp (200 / \mathrm{T})$ \\
\hline $\mathrm{RA} 3 \mathrm{P}+\mathrm{OH}->0.64 \mathrm{OH}+0.36 \mathrm{~A} 3 \mathrm{O} 2+0.64 \mathrm{RCHO}$ & $5.18 \mathrm{E}-12 * \exp (200 / \mathrm{T})$ \\
\hline $\mathrm{RB} 3 \mathrm{P}+\mathrm{OH}->0.791 \mathrm{OH}+0.209 \mathrm{~B} 3 \mathrm{O} 2+0.791 \mathrm{ACET}$ & $8.78 \mathrm{E}-12 * \exp (200 / \mathrm{T})$ \\
\hline $\mathrm{R} 4 \mathrm{P}+\mathrm{OH}->0.791 \mathrm{OH}+0.209 \mathrm{R} 4 \mathrm{O} 2+0.791 \mathrm{RCHO}$ & $8.78 \mathrm{E}-12 * \exp (200 / \mathrm{T})$ \\
\hline $\mathrm{RP}+\mathrm{OH}->\mathrm{RCO} 3$ & $6.13 \mathrm{E}-13 * \exp (200 / \mathrm{T})$ \\
\hline $\mathrm{PP}+\mathrm{OH}->0.791 \mathrm{OH}+0.209 \mathrm{PO} 2+0.791 \mathrm{HAC}$ & 8.78E-12*exp $(200 / \mathrm{T})$ \\
\hline $\mathrm{RIP}+\mathrm{OH}->0.387 \mathrm{RIO} 2+0.613 \mathrm{OH}+0.613 \mathrm{HC} 5$ & 4.75E-12*exp $(200 / \mathrm{T})$ \\
\hline $\mathrm{RIP}+\mathrm{OH}->\mathrm{OH}+\mathrm{IEPOX}$ & $1.9 \mathrm{E}-11 * \exp (390 / \mathrm{T})$ \\
\hline IEPOX + OH -> IEPOXOO & $5.78 \mathrm{E}-11 * \exp (-400 / \mathrm{T})$ \\
\hline $\mathrm{IAP}+\mathrm{OH}->0.654 \mathrm{OH}+0.654 \mathrm{DHMOB}+0.346 \mathrm{HC} 5 \mathrm{OO}$ & $5.31 \mathrm{E}-12 * \exp (200 / \mathrm{T})$ \\
\hline $\mathrm{VRP}+\mathrm{OH}->0.791 \mathrm{OH}+0.791 \mathrm{MEK}+0.209 \mathrm{VRO} 2$ & 8.78E-12*exp $(200 / \mathrm{T})$ \\
\hline $\mathrm{MRP}+\mathrm{OH}->\mathrm{MRO} 2$ & $1.84 \mathrm{E}-12 * \exp (200 / \mathrm{T})$ \\
\hline $\mathrm{MRP}+\mathrm{OH}->(\mathrm{CO} 2)+\mathrm{HAC}+\mathrm{OH}$ & $4.40 \mathrm{E}-12 * \exp (380 / \mathrm{T})$ \\
\hline $\mathrm{MAOP}+\mathrm{OH}->$ MAO3 & $6.13 \mathrm{E}-13 * \exp (200 / \mathrm{T})$ \\
\hline $\mathrm{MAOP}+\mathrm{OH}->\mathrm{MAOPO} 2$ & $3.60 \mathrm{E}-12 * \exp (380 / \mathrm{T})$ \\
\hline $\mathrm{OH}+\mathrm{MAP}$-> 1.0MCO3 & $6.13 \mathrm{E}-13 * \exp (200 / \mathrm{T})$ \\
\hline $\mathrm{HC} 5+\mathrm{OH}->\mathrm{HC} 5 \mathrm{OO}$ & $3.35 \mathrm{E}-11 * \exp (380 / \mathrm{T})$ \\
\hline ISOPND + OH -> ISOPNDO2 & $2.64 \mathrm{E}-11 * \exp (380 / \mathrm{T})$ \\
\hline ISOPNB + OH -> ISOPNBO2 & $3.61 \mathrm{E}-12 * \exp (380 / \mathrm{T})$ \\
\hline $\begin{array}{l}\text { ISNP + OH }->0.612 \mathrm{OH}+0.612 \mathrm{R} 4 \mathrm{~N} 1+0.193 \mathrm{ISOPNBO} 2+ \\
0.193 \mathrm{ISOPNDO} 2\end{array}$ & $4.75 \mathrm{E}-12 * \exp (200 / \mathrm{T})$ \\
\hline $\begin{array}{l}\text { MVKN + OH -> 0.650HCOOH + NO3 + 0.650MGLY + 0.350CH2O + } \\
\text { 0.350PYAC }\end{array}$ & $1.5 \mathrm{E}-12 * \exp (380 / \mathrm{T})$ \\
\hline MACRN + OH -> 1.0MACRNO2 & $1.39 \mathrm{E}-11 * \exp (380 / \mathrm{T})$ \\
\hline $\mathrm{DHMOB}+\mathrm{OH}->1.5 \mathrm{CO}+1.0 \mathrm{HO} 2+0.5 \mathrm{HAC}+0.5 \mathrm{MEK}$ & $2.52 \mathrm{E}-11 * \exp (410 / \mathrm{T})$ \\
\hline $\mathrm{MOBA}+\mathrm{OH}->$ MOBAOO & $2.79 \mathrm{E}-11 * \exp (380 / \mathrm{T})$ \\
\hline $\mathrm{ETHLN}+\mathrm{OH}->\mathrm{CH} 2 \mathrm{O}+(\mathrm{CO} 2)+\mathrm{NO} 2$ & $1.00 \mathrm{E}-11$ \\
\hline $\mathrm{PROPNN}+\mathrm{OH}->\mathrm{NO} 2+\mathrm{MGLY}$ & $1.00 \mathrm{E}-15$ \\
\hline $\mathrm{ATOOH}+\mathrm{OH}->\mathrm{ATO} 2+\mathrm{H} 2 \mathrm{O}$ & $2.66 \mathrm{E}-12 * \exp (200 / \mathrm{T})$ \\
\hline $\mathrm{ATOOH}+\mathrm{OH}->\mathrm{MGLY}+\mathrm{OH}+\mathrm{H} 2 \mathrm{O}$ & $1.14 \mathrm{E}-12 * \exp (200 / \mathrm{T})$ \\
\hline $\mathrm{R} 4 \mathrm{~N} 2+\mathrm{OH}$-> R4N1 + H2O & $1.60 \mathrm{E}-12$ \\
\hline $\begin{array}{l}\text { RIO2 + NO -> 0.883NO2 + 0.783HO2 + 0.660CH2O + 0.400MVK + } \\
0.260 \mathrm{MACR}+0.070 \mathrm{ISOPND}+0.047 \mathrm{ISOPNB}+0.123 \mathrm{HC} 5+\end{array}$ & $2.70 \mathrm{E}-12 * \exp (350 / \mathrm{T})^{\mathrm{a}}$ \\
\hline
\end{tabular}




\begin{tabular}{|c|c|}
\hline \multicolumn{2}{|l|}{$0.1 \mathrm{DIBOO}$} \\
\hline $\begin{array}{l}\text { VRO2 + NO -> 0.88NO2 + 0.35HO2 + 0.35CH2O + 0.53MCO3 + } \\
0.53 G L Y C+0.35 \mathrm{MGLY}+0.12 \mathrm{MVKN}\end{array}$ & $2.70 \mathrm{E}-12 * \exp (350 / \mathrm{T})$ \\
\hline $\begin{array}{l}\text { MRO2 + NO -> 0.85NO2 + 0.85HO2 + 0.122MGLY + 0.728HAC + } \\
0.728 \mathrm{CO}+0.122 \mathrm{CH} 2 \mathrm{O}+0.15 \mathrm{MACRN}\end{array}$ & $2.70 \mathrm{E}-12 * \exp (350 / \mathrm{T})$ \\
\hline $\begin{array}{l}\text { MAN2 + NO -> 1.5NO2 + 0.5CH2O + 0.5MGLY + 0.5PROPNN + } \\
0.5 \mathrm{CO}+0.5 \mathrm{OH}\end{array}$ & $2.70 \mathrm{E}-12 * \exp (350 / \mathrm{T})$ \\
\hline $\begin{array}{l}\text { IEPOXOO + NO }->0.725 \mathrm{HAC}+0.275 \mathrm{GLYC}+0.275 \mathrm{GLYX}+ \\
0.275 \mathrm{MGLY}+0.125 \mathrm{OH}+0.825 \mathrm{HO} 2+(0.200 \mathrm{CO} 2)+0.375 \mathrm{CH} 2 \mathrm{O}+ \\
0.074 \mathrm{HCOOH}+0.251 \mathrm{CO}+\mathrm{NO} 2\end{array}$ & $2.70 \mathrm{E}-12 * \exp (350 / \mathrm{T})$ \\
\hline $\mathrm{MAOPO} 2+\mathrm{NO}->1.0 \mathrm{HAC}+(1.0 \mathrm{CO} 2)+1.0 \mathrm{OH}+1.0 \mathrm{NO} 2$ & $2.48 \mathrm{E}-12$ \\
\hline MAOPO2 + NO -> 1.0HNO3 & $2.24 \mathrm{E}-13$ \\
\hline $\begin{array}{l}\mathrm{HC} 5 \mathrm{OO}+\mathrm{NO}->\mathrm{NO} 2+0.216 \mathrm{GLYX}+0.234 \mathrm{MGLY}+0.234 \mathrm{GLYC}+ \\
0.216 \mathrm{HAC}+0.290 \mathrm{DHMOB}+0.170 \mathrm{MOBA}+0.090 \mathrm{RCHO}+\mathrm{HO} 2+ \\
0.090 \mathrm{CO}\end{array}$ & $2.48 \mathrm{E}-12$ \\
\hline $\mathrm{HC} 5 \mathrm{OO}+\mathrm{NO}->\mathrm{HNO} 3$ & $3.42 \mathrm{E}-13$ \\
\hline $\begin{array}{l}\text { ISOPNDO2 + NO -> 0.070MACRN + 0.310HCOOH + 0.440HAC + } \\
0.130 \mathrm{ETHLN}+0.650 \mathrm{CH} 2 \mathrm{O}+1.34 \mathrm{NO} 2+0.150 \mathrm{GLYC}+0.310 \mathrm{NO} 3+ \\
0.150 \mathrm{PROPNN}+0.340 \mathrm{MEK}+0.350 \mathrm{HO} 2\end{array}$ & $2.48 \mathrm{E}-12$ \\
\hline ISOPNDO2 + NO -> HNO3 & $3.42 \mathrm{E}-13$ \\
\hline $\begin{array}{l}\text { ISOPNBO2 + NO -> 0.6GLYC + 0.6HAC + 0.4CH2O + 1.6NO2 + } \\
0.26 \mathrm{MACRN}+0.4 \mathrm{HO} 2+0.14 \mathrm{MVKN}\end{array}$ & $2.48 \mathrm{E}-12$ \\
\hline ISOPNBO2 + NO -> HNO3 & $3.42 \mathrm{E}-13$ \\
\hline $\begin{array}{l}\text { MACRNO2 + NO -> 0.08ACTA + 0.08CH2O + 0.15NO3 + } \\
0.07 \mathrm{HCOOH}+0.070 \mathrm{MGLY}+0.850 \mathrm{HAC}+0.85 \mathrm{NO} 2+(0.93 \mathrm{CO} 2)+ \\
1.0 \mathrm{NO} 2\end{array}$ & $2.7 \mathrm{E}-12 * \exp (350 / \mathrm{T})$ \\
\hline $\begin{array}{l}\text { DIBOO + NO -> HO2 + NO2 + 0.520GLYC + 0.520MGLY + } \\
0.480 H A C+0.480 G L Y X\end{array}$ & $2.48 \mathrm{E}-12$ \\
\hline $\mathrm{DIBOO}+\mathrm{NO}->\mathrm{HNO}$ & $3.42 \mathrm{E}-13$ \\
\hline $\mathrm{MOBAOO}+\mathrm{NO}->\mathrm{RCHO}+(\mathrm{CO} 2)+\mathrm{HO} 2+\mathrm{NO} 2$ & 2.48E-12 \\
\hline MOBAOO + NO -> HNO3 & $3.42 \mathrm{E}-13$ \\
\hline $\begin{array}{l}\text { MAN2 + NO -> 1.5NO2 + 0.5CH2O + 0.5MGLY + 0.5PROPNN + } \\
0.5 \mathrm{CO}+0.5 \mathrm{OH}\end{array}$ & $2.70 \mathrm{E}-12 * \exp (350 / \mathrm{T})$ \\
\hline $\mathrm{MCO} 3+\mathrm{NO}->\mathrm{MO} 2+\mathrm{NO} 2+(\mathrm{CO} 2)$ & $8.10 \mathrm{E}-12 * \exp (270 / \mathrm{T})$ \\
\hline $\mathrm{RCO} 3+\mathrm{NO}->\mathrm{NO} 2+\mathrm{ETO} 2$ & $6.70 \mathrm{E}-12 * \exp (340 / \mathrm{T})$ \\
\hline $\begin{array}{l}\mathrm{MAO} 3+\mathrm{NO}->\mathrm{NO} 2+0.5 \mathrm{CH} 2 \mathrm{O}+0.5 \mathrm{CO}+(\mathrm{CO} 2)+0.5 \mathrm{MO} 2+ \\
0.5 \mathrm{MCO} 3\end{array}$ & $6.70 \mathrm{E}-12 * \exp (340 / \mathrm{T})$ \\
\hline $\mathrm{ATO} 2+\mathrm{NO}->0.96 \mathrm{NO} 2+0.960 \mathrm{CH} 2 \mathrm{O}+0.960 \mathrm{MCO} 3+0.04 \mathrm{R} 4 \mathrm{~N} 2$ & $2.80 \mathrm{E}-12 * \exp (300 / \mathrm{T})$ \\
\hline $\begin{array}{l}\text { RIO2 + HO2 -> 0.88RIP + 0.12OH + 0.047MACR + 0.073MVK + } \\
0.12 \mathrm{HO} 2+0.12 \mathrm{CH} 2 \mathrm{O}\end{array}$ & $2.06 \mathrm{E}-13 * \exp (1300 / \mathrm{T})$ \\
\hline $\begin{array}{l}\text { VRO2 + HO2 }->0.1 \mathrm{VRP}+0.68 \mathrm{OH}+0.578 \mathrm{GLYC}+0.578 \mathrm{MCO} 3+ \\
0.187 \mathrm{MEK}+0.102 \mathrm{HO} 2+0.102 \mathrm{CH} 2 \mathrm{O}+0.102 \mathrm{MGLY}+0.033 \mathrm{RCHO}\end{array}$ & $1.82 \mathrm{E}-13 * \exp (1300 / \mathrm{T})$ \\
\hline $\mathrm{MRO} 2+\mathrm{HO} 2->1.0 \mathrm{MRP}$ & $1.82 \mathrm{E}-13 * \exp (1300 / \mathrm{T})$ \\
\hline $\begin{array}{l}\text { MAN2 + HO2 -> 0.075PROPNN + 0.075CO + 0.075HO2 + } \\
0.075 \mathrm{MGLY}+0.075 \mathrm{CH} 2 \mathrm{O}+0.075 \mathrm{NO} 2+0.15 \mathrm{OH}+0.85 \mathrm{ISNP}\end{array}$ & $1.82 \mathrm{E}-13 * \exp (1300 / \mathrm{T})$ \\
\hline $\begin{array}{l}\text { IEPOXOO + HO2 -> 0.725HAC + 0.275GLYC + 0.275GLYX + } \\
0.275 \mathrm{MGLY}+1.125 \mathrm{OH}+0.825 \mathrm{HO} 2+(0.200 \mathrm{CO} 2)+0.375 \mathrm{CH} 2 \mathrm{O}+ \\
0.074 \mathrm{HCOOH}+0.251 \mathrm{CO}\end{array}$ & $2.06 \mathrm{E}-13 * \exp (1300 / \mathrm{T})$ \\
\hline $\begin{array}{l}\mathrm{DIBOO}+\mathrm{HO} 2->0.15 \mathrm{HO}+0.15 \mathrm{OH}+0.078 \mathrm{GLYC}+0.078 \mathrm{MGLY}+ \\
0.072 \mathrm{HAC}+0.072 \mathrm{GLYX}+0.85 \mathrm{R} 4 \mathrm{P}\end{array}$ & $2.06 \mathrm{E}-13 * \exp (1300 / \mathrm{T})$ \\
\hline $\mathrm{MAOPO} 2+\mathrm{HO} 2->1.0 \mathrm{HAC}+(1.0 \mathrm{CO} 2)+2.0 \mathrm{OH}$ & $1.82 \mathrm{E}-13 * \exp (1300 / \mathrm{T})$ \\
\hline
\end{tabular}




\begin{tabular}{|c|c|}
\hline $\mathrm{HC} 5 \mathrm{OO}+\mathrm{HO} 2->0.1 \mathrm{IAP}+0.9 \mathrm{OH}+0.9 \mathrm{MGLY}+0.9 \mathrm{GLYC}+0.9 \mathrm{HO} 2$ & $2.06 \mathrm{E}-13^{*} \exp (1300 / \mathrm{T})$ \\
\hline ISOPNDO2 + HO2 -> 0.5OH + 0.035MACRN + $0.155 \mathrm{HCOOH}+$ & \\
\hline $\begin{array}{l}0.22 \mathrm{HAC}+0.065 \mathrm{ETHLN}+0.325 \mathrm{CH} 2 \mathrm{O}+0.170 \mathrm{NO} 2+0.075 \mathrm{GLYC}+ \\
0.155 \mathrm{NO} 3+0.075 \mathrm{PROPNN}+0.170 \mathrm{MEK}+0.175 \mathrm{HO} 2+0.5 \mathrm{ISNP}\end{array}$ & 2.06E-13*exp $(1300 / \mathrm{T})$ \\
\hline $\begin{array}{l}\text { ISOPNBO2 + HO2 -> 0.3GLYC + 0.3HAC + 0.2CH2O + 0.13MACRN } \\
+0.07 \mathrm{MVKN}+0.3 \mathrm{NO} 2+0.2 \mathrm{HO} 2+0.5 \mathrm{OH}+0.5 \mathrm{ISNP}\end{array}$ & $2.06 \mathrm{E}-13 * \exp (1300 / \mathrm{T})$ \\
\hline $\begin{array}{l}\text { MACRNO2 + HO2 -> 0.08ACTA + 0.08CH2O + 0.15NO3 + } \\
0.07 \mathrm{HCOOH}+0.07 \mathrm{MGLY}+0.85 \mathrm{HAC}+0.85 \mathrm{NO} 2+(0.93 \mathrm{CO} 2)+ \\
1.0 \mathrm{OH}\end{array}$ & $1.82 \mathrm{E}-13 * \exp (1300 / \mathrm{T})$ \\
\hline $\begin{array}{l}\text { MOBAOO + HO2 }->0.15 \mathrm{OH}+0.15 \mathrm{HO} 2+0.15 \mathrm{RCHO}+(0.15 \mathrm{CO} 2)+ \\
0.85 \mathrm{R} 4 \mathrm{P}\end{array}$ & $2.06 \mathrm{E}-13 * \exp (1300 / \mathrm{T})$ \\
\hline $\begin{array}{l}\mathrm{MCO} 3+\mathrm{HO} 2->0.15 \mathrm{ACTA}+0.15 \mathrm{O} 3+0.44 \mathrm{OH}+0.44 \mathrm{MO} 2+ \\
0.41 \mathrm{MAP}\end{array}$ & $5.20 \mathrm{E}-13 * \exp (980 / \mathrm{T})$ \\
\hline $\begin{array}{l}\text { RCO3 + HO2 -> 0.410RP + 0.150RCOOH + } 0.150 \mathrm{O} 3+0.440 \mathrm{OH}+ \\
0.440 \mathrm{ETO} 2\end{array}$ & 4.30E-13*exp $(1040 / \mathrm{T})$ \\
\hline $\mathrm{ATO} 2+\mathrm{HO} 2->0.15 \mathrm{MCO} 3+0.15 \mathrm{OH}+0.15 \mathrm{CH} 2 \mathrm{O}+0.85 \mathrm{ATOOH}$ & $8.60 \mathrm{E}-13 * \exp (700 / \mathrm{T})$ \\
\hline $\mathrm{KO} 2+\mathrm{HO} 2->0.15 \mathrm{OH}+0.15 \mathrm{ALD} 2+0.15 \mathrm{MCO} 3+0.85 \mathrm{ATOOH}$ & $1.82 \mathrm{E}-13 * \exp (1300 / \mathrm{T})$ \\
\hline $\begin{array}{l}\mathrm{MAO} 3+\mathrm{HO} 2->0.44 \mathrm{OH}+0.15 \mathrm{O} 3+0.59 \mathrm{CH} 2 \mathrm{O}+0.39 \mathrm{MO} 2+ \\
0.41 \mathrm{MAOP}+0.39 \mathrm{CO}\end{array}$ & 4.30E-13*exp $(1040 / \mathrm{T})$ \\
\hline $\begin{array}{l}\mathrm{RIO} 2+\mathrm{MO} 2->1.1 \mathrm{HO} 2+1.22 \mathrm{CH} 2 \mathrm{O}+0.280 \mathrm{MVK}+0.180 \mathrm{MACR}+ \\
0.3 \mathrm{HC} 5+0.24 \mathrm{MOH}+0.24 \mathrm{ROH}\end{array}$ & 8.37E-14 \\
\hline $\begin{array}{l}\mathrm{HC} 5 \mathrm{OO}+\mathrm{MO} 2->0.50 \mathrm{HO} 2+0.33 \mathrm{CO}+0.09 \mathrm{H} 2+0.18 \mathrm{HAC}+ \\
0.13 \mathrm{GLYC}+0.29 \mathrm{MGLY}+0.25 \mathrm{MEK}+0.95 \mathrm{CH} 2 \mathrm{O}+0.25 \mathrm{MOH}+ \\
0.25 \mathrm{ROH}+0.5 \mathrm{HO} 2\end{array}$ & 8.37E-14 \\
\hline $\begin{array}{l}\text { MRO2 + MO2 - 0.595HAC + 0.255MGLY + 0.595CO + 1.255CH2O } \\
+1.7 \mathrm{HO} 2+0.150 \mathrm{ROH}\end{array}$ & 8.37E-14 \\
\hline $\begin{array}{l}\mathrm{VRO} 2+\mathrm{MO} 2->0.14 \mathrm{HO} 2+0.14 \mathrm{CH} 2 \mathrm{O}+0.36 \mathrm{MCO}+0.36 \mathrm{GLYC}+ \\
0.14 \mathrm{MGLY}+0.25 \mathrm{MEK}+0.75 \mathrm{CH} 2 \mathrm{O}+0.25 \mathrm{MOH}+0.25 \mathrm{ROH}+ \\
0.5 \mathrm{HO} 2\end{array}$ & 8.37E-14 \\
\hline $\begin{array}{l}\text { MAN2 + MO2 -> 0.375PROPNN + 0.375CO + 0.375HO2 + } \\
0.375 \mathrm{MGLY}+0.375 \mathrm{CH} 2 \mathrm{O}+0.375 \mathrm{NO} 2+0.250 \mathrm{CH} 2 \mathrm{O}+0.250 \mathrm{R} 4 \mathrm{~N} 2\end{array}$ & 8.37E-14 \\
\hline $\begin{array}{l}\mathrm{MAOPO} 2+\mathrm{MO} 2->0.7 \mathrm{HAC}+(0.7 \mathrm{CO} 2)+0.7 \mathrm{OH}+1.0 \mathrm{CH} 2 \mathrm{O}+ \\
0.7 \mathrm{HO} 2+0.3 \mathrm{ROH}\end{array}$ & 8.37E-14 \\
\hline $\begin{array}{l}2 \mathrm{RIO} 2 \text {-> } 1.28 \mathrm{HO} 2+0.92 \mathrm{CH} 2 \mathrm{O}+0.56 \mathrm{MVK}+0.36 \mathrm{MACR}+0.48 \mathrm{ROH} \\
+0.5 \mathrm{HC} 5\end{array}$ & $1.54 \mathrm{E}-13$ \\
\hline $2 \mathrm{MAOPO} 2->2.0 \mathrm{HAC}+(2.0 \mathrm{CO} 2)+2.0 \mathrm{OH}$ & 8.37E-14 \\
\hline $\mathrm{MCO} 3+\mathrm{MO} 2->\mathrm{CH} 2 \mathrm{O}+\mathrm{MO} 2+\mathrm{HO} 2$ & $1.80 \mathrm{E}-12 * \exp (500 / \mathrm{T})$ \\
\hline $\mathrm{MCO} 3+\mathrm{MO} 2->\mathrm{ACTA}+\mathrm{CH} 2 \mathrm{O}$ & $2.00 \mathrm{E}-13 * \exp (500 / \mathrm{T})$ \\
\hline $\mathrm{RCO} 3+\mathrm{MO} 2->\mathrm{CH} 2 \mathrm{O}+\mathrm{HO} 2+\mathrm{ETO} 2$ & $1.68 \mathrm{E}-12 * \exp (500 / \mathrm{T})$ \\
\hline $\mathrm{RCO} 3+\mathrm{MO} 2->\mathrm{RCOOH}+\mathrm{CH} 2 \mathrm{O}$ & $1.87 \mathrm{E}-13 * \exp (500 / \mathrm{T})$ \\
\hline $\mathrm{MAO} 3+\mathrm{MO} 2->\mathrm{CH} 2 \mathrm{O}+\mathrm{HO} 2+\mathrm{CH} 2 \mathrm{O}+\mathrm{MCO} 3$ & $1.68 \mathrm{E}-12 * \exp (500 / \mathrm{T})$ \\
\hline $\mathrm{MAO} 3+\mathrm{MO} 2$-> RCOOH + CH2O & $1.87 \mathrm{E}-13 * \exp (500 / \mathrm{T})$ \\
\hline $\mathrm{MAOPO} 2+\mathrm{MCO} 3->1.0 \mathrm{HAC}+(2.0 \mathrm{CO} 2)+\mathrm{OH}+\mathrm{MO} 2$ & $1.68 \mathrm{E}-12 * \exp (500 / \mathrm{T})$ \\
\hline MAOPO2 + MCO3 -> 1.0ACTA + 1.0MEK & $1.87 \mathrm{E}-13 * \exp (500 / \mathrm{T})$ \\
\hline $\begin{array}{l}\mathrm{R} 4 \mathrm{O} 2+\mathrm{MCO} 3->\mathrm{MO} 2+0.32 \mathrm{ACET}+0.19 \mathrm{MEK}+0.27 \mathrm{HO} 2+ \\
0.32 \mathrm{ALD} 2+0.13 \mathrm{RCHO}+0.05 \mathrm{~A} 3 \mathrm{O} 2+0.18 \mathrm{~B} 3 \mathrm{O} 2+0.32 \mathrm{ETO} 2\end{array}$ & $1.68 \mathrm{E}-12 * \exp (500 / \mathrm{T})$ \\
\hline $\mathrm{R} 4 \mathrm{O} 2+\mathrm{MCO} 3->1.0 \mathrm{ACTA}+1.0 \mathrm{MEK}$ & $1.87 \mathrm{E}-13 * \exp (500 / \mathrm{T})$ \\
\hline $\mathrm{ATO} 2+\mathrm{MCO} 3->\mathrm{MCO} 3+\mathrm{CH} 2 \mathrm{O}+\mathrm{MO} 2$ & $1.68 \mathrm{E}-12 * \exp (500 / \mathrm{T})$ \\
\hline ATO2 + MCO3 -> MGLY + ACTA & $1.87 \mathrm{E}-13 * \exp (500 / \mathrm{T})$ \\
\hline $\begin{array}{l}\text { HC5OO + MCO3 -> 0.216GLYX + 0.234MGLY + 0.234GLYC + } \\
0.216 \mathrm{HAC}+0.29 \mathrm{DHMOB}+0.17 \mathrm{MOBA}+0.09 \mathrm{RCHO}+\mathrm{HO} 2+\end{array}$ & $1.68 \mathrm{E}-12 * \exp (500 / \mathrm{T})$ \\
\hline
\end{tabular}




\begin{tabular}{|c|c|}
\hline \multicolumn{2}{|l|}{$0.09 \mathrm{CO}+\mathrm{MO} 2$} \\
\hline HC5OO + MCO3 -> MEK + ACTA & $1.87 \mathrm{E}-13 * \exp (500 / \mathrm{T})$ \\
\hline $\begin{array}{l}\mathrm{VRO} 2+\mathrm{MCO} 3->0.4 \mathrm{HO} 2+0.4 \mathrm{CH} 2 \mathrm{O}+0.6 \mathrm{MCO} 3+0.6 \mathrm{GLYC}+ \\
0.4 \mathrm{MGLY}+1.0 \mathrm{MO} 2\end{array}$ & $1.68 \mathrm{E}-12 * \exp (500 / \mathrm{T})$ \\
\hline VRO2 + MCO3 -> MEK + ACTA & $1.87 \mathrm{E}-13 * \exp (500 / \mathrm{T})$ \\
\hline $\begin{array}{l}\text { MRO2 + MCO3 -> 0.850HO2 + 0.143MGLY + 0.857HAC + 0.857CO } \\
+0.143 \mathrm{CH} 2 \mathrm{O}+1.0 \mathrm{MO} 2\end{array}$ & $1.68 \mathrm{E}-12 * \exp (500 / \mathrm{T})$ \\
\hline MRO2 + MCO3 -> MEK + ACTA & $1.87 \mathrm{E}-13 * \exp (500 / \mathrm{T})$ \\
\hline $\begin{array}{l}\text { MAN2 + MCO3 -> 0.5PROPNN + 0.5CO + 0.5HO2 + 0.5MGLY + } \\
0.5 \mathrm{CH} 2 \mathrm{O}+0.5 \mathrm{NO} 2+(\mathrm{CO} 2)+\mathrm{MO} 2\end{array}$ & $1.68 \mathrm{E}-12 * \exp (500 / \mathrm{T})$ \\
\hline MAN2 + MCO3 -> RCHO + ACTA + NO2 & $1.87 \mathrm{E}-13 * \exp (500 / \mathrm{T})$ \\
\hline $\begin{array}{l}\text { RIO2 + MCO3 -> 0.887HO2 + 0.747CH2O + 0.453MVK + } \\
0.294 \mathrm{MACR}+0.140 \mathrm{HC} 5+0.113 \mathrm{DIBOO}+(\mathrm{CO} 2)+\mathrm{MO} 2\end{array}$ & $1.68 \mathrm{E}-12 * \exp (500 / \mathrm{T})$ \\
\hline $\mathrm{RIO} 2+\mathrm{MCO} 3$-> MEK + ACTA & $1.87 \mathrm{E}-13 * \exp (500 / \mathrm{T})$ \\
\hline MCO3 + MCO3 -> 2MO2 & $2.50 \mathrm{E}-12 * \exp (500 / \mathrm{T})$ \\
\hline $\mathrm{RCO} 3+\mathrm{MCO} 3->\mathrm{MO} 2+\mathrm{ETO} 2$ & $2.50 \mathrm{E}-12 * \exp (500 / \mathrm{T})$ \\
\hline $\mathrm{MAO} 3+\mathrm{MCO} 3->\mathrm{MO} 2+\mathrm{MCO} 3+\mathrm{CH} 2 \mathrm{O}$ & $2.50 \mathrm{E}-12 * \exp (500 / \mathrm{T})$ \\
\hline $\mathrm{MCO} 3+\mathrm{NO} 2$-> PAN & 8.59E-12 \\
\hline $\mathrm{PAN}->\mathrm{MCO} 3+\mathrm{NO} 2$ & 5.73E-04 \\
\hline $\mathrm{RCO} 3+\mathrm{NO} 2$-> PPN & 7.40E-12 \\
\hline $\mathrm{PPN}->\mathrm{RCO} 3+\mathrm{NO} 2$ & 4.44E-04 \\
\hline $\mathrm{MAO} 3+\mathrm{NO} 2$-> PMN & $7.40 \mathrm{E}-12$ \\
\hline $\mathrm{PMN}$-> MAO3 + NO2 & 4.44E-04 \\
\hline MACRNO2 + NO2 -> PMNN & 7.40E-12 \\
\hline PMNN -> MACRNO2 + NO2 & 4.44E-04 \\
\hline $\begin{array}{l}\mathrm{ISOP}+\mathrm{O} 3 \text {-> 0.244MVK + 0.325MACR + 0.845CH2O + 0.110H2O2 + } \\
0.522 \mathrm{CO}+0.204 \mathrm{HCOOH}+0.199 \mathrm{MCO}+0.026 \mathrm{HO} 2+0.270 \mathrm{OH}+ \\
0.128 \mathrm{PRPE}+0.051 \mathrm{MO} 2\end{array}$ & $1.00 \mathrm{E}-14 * \exp (-1970 / \mathrm{T})$ \\
\hline $\begin{array}{l}\mathrm{MVK}+\mathrm{O} 3->0.202 \mathrm{OH}+0.202 \mathrm{HO} 2+0.352 \mathrm{HCOOH}+0.535 \mathrm{CO}+ \\
0.050 \mathrm{ALD} 2+0.950 \mathrm{MGLY}+0.050 \mathrm{CH} 2 \mathrm{O}\end{array}$ & 8.50E-16*exp(-1520/T) \\
\hline $\begin{array}{l}\text { MACR + O3 }->0.261 \mathrm{OH}+0.202 \mathrm{HO} 2+0.326 \mathrm{HCOOH}+0.569 \mathrm{CO}+ \\
0.880 \mathrm{MGLY}+0.120 \mathrm{CH} 2 \mathrm{O}\end{array}$ & $1.40 \mathrm{E}-15^{*} \exp (-2100 / \mathrm{T})$ \\
\hline $\begin{array}{l}\mathrm{HC} 5+\mathrm{O} 3->0.6 \mathrm{MGLY}+0.1 \mathrm{OH}+0.12 \mathrm{CH} 2 \mathrm{O}+0.28 \mathrm{GLYC}+0.3 \mathrm{O} 3+ \\
0.4 \mathrm{CO}+0.2 \mathrm{H} 2+0.2 \mathrm{HAC}+0.2 \mathrm{HCOOH}\end{array}$ & 6.16E-15*exp $(-1814 / \mathrm{T})$ \\
\hline ISOPNB + O3 -> 0.610MVKN + 0.390MACRN + 0.27OH + CH2O & $1.06 \mathrm{E}-16$ \\
\hline $\begin{array}{l}\text { ISOPND + O3 -> 0.5PROPNN + 0.5ETHLN + 0.27OH + 0.5GLYC + } \\
0.5 \mathrm{HAC}\end{array}$ & 5.30E-17 \\
\hline $\mathrm{MOBA}+\mathrm{O} 3->\mathrm{OH}+\mathrm{HO} 2+(\mathrm{CO} 2)+\mathrm{MEK}$ & 2.00E-17 \\
\hline $\mathrm{PMN}+\mathrm{O} 3$-> NO2 + 0.6CH2O + HO2 & 8.20E-18 \\
\hline $\begin{array}{l}\text { RIO2 -> 2.0HO2 + 1.0CH2O + 0.5MGLY + 0.5GLYC + 0.5GLYX + } \\
0.500 \mathrm{HAC}+1.0 \mathrm{OH}\end{array}$ & 4.07E+08*exp(-7694/T) \\
\hline $\mathrm{MRO} 2->1.0 \mathrm{CO}+1.0 \mathrm{HAC}+1.0 \mathrm{OH}$ & $2.90 \mathrm{E}+07 * \exp (-5297 / \mathrm{T})$ \\
\hline $\mathrm{C} 10 \mathrm{H} 16+\mathrm{OH}->$ TERPO2 & $1.20 \mathrm{E}-11 * \exp (444 / \mathrm{T})$ \\
\hline $\mathrm{C} 10 \mathrm{H} 16+\mathrm{O} 3->.7 \mathrm{OH}+\mathrm{MVK}+\mathrm{MACR}+\mathrm{HO} 2$ & $1.00 \mathrm{E}-15 * \exp (-732 / \mathrm{T})$ \\
\hline $\mathrm{C} 10 \mathrm{H} 16+\mathrm{NO} 3$-> TERPO2 + NO2 & $1.20 \mathrm{E}-12 * \exp (490 / \mathrm{T})$ \\
\hline TERPO2 + NO -> .1ACET + MVK + MACR + NO2 & $4.20 \mathrm{E}-12 * \exp (180 / \mathrm{T})$ \\
\hline TERPO2 + HO2 -> TERPOOH & $7.50 \mathrm{E}-13 * \exp (700 / \mathrm{T})$ \\
\hline TERPOOH + OH -> TERPO2 & $3.80 \mathrm{E}-12 * \exp (200 / \mathrm{T})$ \\
\hline $\mathrm{O} 3->\mathrm{O} 2+\mathrm{O} 1 \mathrm{D}$ & $2.80 \mathrm{E}-04 * \exp (-1.75 / \cos (\mathrm{x}))$ \\
\hline $\mathrm{O} 3->\mathrm{O} 2+\mathrm{O}$ & $6.76 \mathrm{E}-04 * \exp (-0.30 / \cos (\mathrm{x}))$ \\
\hline
\end{tabular}




\begin{tabular}{|c|c|}
\hline $\mathrm{H} 2 \mathrm{O} 2->2 \mathrm{OH}$ & $2.10 \mathrm{E}-05^{*} \exp (-0.86 / \cos (\mathrm{x}))$ \\
\hline $\mathrm{NO} 2->\mathrm{NO}+\mathrm{O}$ & $1.71 \mathrm{E}-02 * \exp (-0.55 / \cos (\mathrm{x}))$ \\
\hline $\mathrm{NO} 3->\mathrm{NO}+\mathrm{O} 2$ & $3.03 \mathrm{E}-02 * \exp (-0.22 / \cos (\mathrm{x}))$ \\
\hline $\mathrm{NO} 3->\mathrm{NO} 2+\mathrm{O}$ & $2.49 E-01 * \exp (-0.25 / \cos (\mathrm{x}))$ \\
\hline $\mathrm{N} 2 \mathrm{O} 5$-> NO3 + NO + O & $1.02 \mathrm{E}-06 * \exp (-5.50 / \cos (\mathrm{x}))$ \\
\hline $\mathrm{N} 2 \mathrm{O} 5$-> NO3 + NO2 & $1.25 \mathrm{E}-04 * \exp (-0.85 / \cos (\mathrm{x}))$ \\
\hline $\mathrm{HNO} 3$-> OH + NO2 & 2.8E-06*exp(-1.20/cos(x)) \\
\hline $\mathrm{HO} 2 \mathrm{NO} 2$-> HO2 + NO2 & 2.05E-05*exp(-1.20/cos $(\mathrm{x}))$ \\
\hline $\mathrm{CH} 2 \mathrm{O}->2 \mathrm{HO} 2+\mathrm{CO}$ & $1.05 \mathrm{E}-04 * \exp (-1.00 / \cos (\mathrm{x}))$ \\
\hline $\mathrm{CH} 2 \mathrm{O}->\mathrm{H} 2+\mathrm{CO}$ & $1.25 \mathrm{E}-04 * \exp (-0.80 / \cos (\mathrm{x}))$ \\
\hline $\mathrm{MP}->\mathrm{CH} 2 \mathrm{O}+\mathrm{HO} 2+\mathrm{OH}$ & $1.65 \mathrm{E}-05^{*} \exp (-0.88 / \cos (\mathrm{x}))$ \\
\hline $\mathrm{ALD} 2$-> $\mathrm{MO} 2+\mathrm{HO} 2+\mathrm{CO}$ & 2.95E-05*exp(-1.40/cos(x)) \\
\hline ALD2 -> CH4 + CO & $1.25 \mathrm{E}-08 * \exp (-6.00 / \cos (\mathrm{x}))$ \\
\hline $\mathrm{PAN}->0.6 \mathrm{MCO} 3+0.6 \mathrm{NO} 2+0.4 \mathrm{MO} 2+0.4 \mathrm{NO} 3$ & 2.5E-06*exp(-1.00/cos(x)) \\
\hline $\mathrm{RCHO}->\mathrm{ETO} 2+\mathrm{HO} 2+\mathrm{CO}$ & 7.35E-05*exp $(-1.10 / \cos (\mathrm{x}))$ \\
\hline ACET -> MCO3 + MO2 & 3.76E-06*exp(-1.80/cos(x)) \\
\hline MEK -> 0.85MCO3 + 0.85ETO2 + 0.15MO2 + 0.15RCO3 & 3.05E-05*exp(-1.20/cos $(\mathrm{x}))$ \\
\hline $\mathrm{GLYC}->\mathrm{CH} 2 \mathrm{O}+2.0 \mathrm{HO} 2+\mathrm{CO}$ & $2.20 \mathrm{E}-05^{*} \exp (-1.20 / \cos (\mathrm{x}))$ \\
\hline GLYX -> 2.0CO + 2.0HO2 & $1.16 \mathrm{E}-04 * \exp (-0.45 / \cos (\mathrm{x}))$ \\
\hline MGLY -> MCO3 + $\mathrm{CO}+\mathrm{HO} 2$ & $2.60 \mathrm{E}-04 * \exp (-0.60 / \cos (\mathrm{x}))$ \\
\hline MVK -> PRPE + CO & 6.72E-06*exp(-0.77/cos $(\mathrm{x}))$ \\
\hline $\mathrm{MVK}->\mathrm{MCO} 3+\mathrm{CH} 2 \mathrm{O}+\mathrm{CO}+\mathrm{HO} 2$ & 2.24E-06*exp(-0.77/cos $(\mathrm{x}))$ \\
\hline MVK -> MO2 + MAO3 & 2.24E-06*exp(-0.77/cos $(\mathrm{x}))$ \\
\hline MACR -> MAO3 + HO2 & 5.85E-06*exp(-0.60/cos $(\mathrm{x}))$ \\
\hline MACR -> CO + HO2 + CH2O + MCO3 & $5.85 \mathrm{E}-06 * \exp (-0.60 / \cos (\mathrm{x}))$ \\
\hline $\mathrm{HAC}->\mathrm{MCO} 3+\mathrm{CH} 2 \mathrm{O}+\mathrm{HO} 2$ & 4.05E-06*exp(-1.10/cos $(\mathrm{x}))$ \\
\hline $\mathrm{INPN}->\mathrm{OH}+\mathrm{HO} 2+\mathrm{RCHO}+\mathrm{NO} 2$ & $1.65 \mathrm{E}-05^{*} \exp (-0.88 / \cos (\mathrm{x}))$ \\
\hline $\mathrm{PRPN}->\mathrm{OH}+\mathrm{HO} 2+\mathrm{RCHO}+\mathrm{NO} 2$ & 1.65E-05*exp $(-0.88 / \cos (\mathrm{x}))$ \\
\hline $\mathrm{ETP}->\mathrm{OH}+\mathrm{HO} 2+\mathrm{ALD} 2$ & $1.65 \mathrm{E}-05^{*} \exp (-0.88 / \cos (\mathrm{x}))$ \\
\hline $\mathrm{RA} 3 \mathrm{P}->\mathrm{OH}+\mathrm{HO} 2+\mathrm{RCHO}$ & $1.65 \mathrm{E}-05^{*} \exp (-0.88 / \cos (\mathrm{x}))$ \\
\hline RB3P $->\mathrm{OH}+\mathrm{HO} 2+\mathrm{ACET}$ & $1.65 \mathrm{E}-05^{*} \exp (-0.88 / \cos (\mathrm{x}))$ \\
\hline $\mathrm{R} 4 \mathrm{P}->\mathrm{OH}+\mathrm{HO} 2+\mathrm{RCHO}$ & $1.65 \mathrm{E}-05^{*} \exp (-0.88 / \cos (\mathrm{x}))$ \\
\hline $\mathrm{PP}->\mathrm{OH}+\mathrm{HO} 2+\mathrm{ALD} 2+\mathrm{CH} 2 \mathrm{O}$ & $1.65 \mathrm{E}-05^{*} \exp (-0.88 / \cos (\mathrm{x}))$ \\
\hline $\mathrm{RP}->\mathrm{OH}+\mathrm{HO} 2+\mathrm{ALD} 2$ & $1.65 \mathrm{E}-05^{*} \exp (-0.88 / \cos (\mathrm{x}))$ \\
\hline $\begin{array}{l}\text { RIP -> OH + HO2 + 0.710CH2O + 0.425MVK + 0.285MACR + } \\
0.29 \mathrm{HC} 5\end{array}$ & 1.65E-05*exp(-0.88/cos(x)) \\
\hline $\begin{array}{l}\mathrm{IAP}->\mathrm{OH}+\mathrm{HO} 2+0.67 \mathrm{CO}+0.190 \mathrm{H} 2+0.36 \mathrm{HAC}+0.26 \mathrm{GLYC}+ \\
0.580 \mathrm{MGLY}\end{array}$ & $1.65 \mathrm{E}-05^{*} \exp (-0.88 / \cos (\mathrm{x}))$ \\
\hline $\mathrm{ISNP}->\mathrm{OH}+\mathrm{HO} 2+\mathrm{RCHO}+\mathrm{NO} 2$ & 1.65E-05*exp $(-0.88 / \cos (\mathrm{x}))$ \\
\hline $\mathrm{VRP}->\mathrm{OH}+0.3 \mathrm{HO} 2+0.3 \mathrm{CH} 2 \mathrm{O}+0.7 \mathrm{MCO} 3+0.7 \mathrm{GLYC}+0.3 \mathrm{MGLY}$ & $1.65 \mathrm{E}-05^{*} \exp (-0.88 / \cos (\mathrm{x}))$ \\
\hline $\mathrm{MRP}->\mathrm{OH}+\mathrm{HO} 2+\mathrm{HAC}+\mathrm{CO}+\mathrm{CH} 2 \mathrm{O}$ & $1.65 \mathrm{E}-05^{*} \exp (-0.88 / \cos (\mathrm{x}))$ \\
\hline $\mathrm{MAOP}->\mathrm{OH}+\mathrm{CH} 2 \mathrm{O}+\mathrm{MCO} 3$ & $1.65 \mathrm{E}-05 * \exp (-0.88 / \cos (\mathrm{x}))$ \\
\hline $\begin{array}{l}\text { R4N2 -> NO2 + 0.320ACET + 0.190MEK + 0.180MO2 + 0.270HO2 + } \\
0.320 \mathrm{ALD} 2+0.130 \mathrm{RCHO}+0.050 \mathrm{~A} 3 \mathrm{O} 2+0.180 \mathrm{~B} 3 \mathrm{O} 2+0.320 \mathrm{ETO} 2\end{array}$ & 3.94E-06*exp(-1.20/cos $(\mathrm{x}))$ \\
\hline $\mathrm{MAP}->\mathrm{OH}+\mathrm{MO} 2$ & $1.65 \mathrm{E}-05^{*} \exp (-0.88 / \cos (\mathrm{x}))$ \\
\hline MACRN -> NO2 + HAC + MGLY + 0.5CH2O + HO2 + 0.5CO & $2.50 \mathrm{E}-06 * \exp (-1.00 / \cos (\mathrm{x}))$ \\
\hline MVKN -> GLYC + NO2 + MCO3 & $2.50 \mathrm{E}-06 * \exp (-1.00 / \cos (\mathrm{x}))$ \\
\hline ISOPNB -> HC5 + NO2 + HO2 & 2.50E-06*exp(-1.00/cos $(\mathrm{x}))$ \\
\hline
\end{tabular}




\begin{tabular}{ll}
\hline ISOPND -> HC5 + NO2 + HO2 & $2.50 \mathrm{E}-06^{*} \exp (-1.00 / \cos (\mathrm{x}))$ \\
PROPNN -> CH2O + NO2 + CO + MO2 & $2.50 \mathrm{E}-06 * \exp (-1.00 / \cos (\mathrm{x}))$ \\
ATOOH -> OH + CH2O + MCO3 & $1.65 \mathrm{E}-05^{*} \exp (-0.88 / \cos (\mathrm{x}))$ \\
\hline
\end{tabular}

1

2 a The stoichiometric coefficient is in accordance with 12\% ISOPN yield.

3

4

5

6

7

8

9

10

11

12

13

14

15

16

17

18

19

20

21

22

23

24 
3 Table S4. The relationship between solar zenith angle (sza) and photolysis rates $(\mathrm{j}=$ $\left.4 \mathrm{a}^{*} \exp (\mathrm{b} / \cos (\mathrm{sza}))\right)$, which are obtained from the TUV radiation model. Adjusted $\mathrm{R}^{2}$ indicates 5 the adjusted correlation coefficient for each curve fitting result. The last two columns show 6 the photolysis rates obtained from TUV simulations and NCAR C-130 observations at 10:37 7 CST (which corresponds to sza of 0.32), respectively. J-value observations onboard NCAR C8130 over the AABC and SEARCH sites are available on 06/14/2013. The j-values collected 9 below pressure altitude of $1000 \mathrm{~m}$ while over the two ground sites are averaged and used here 10 (sampling time ranges between 10:02 CST and 11:11 CST with middle sampling time at 11 10:37 CST). NA indicates no observations were available for the specific reaction.

\begin{tabular}{|c|c|c|c|c|c|c|}
\hline Number & Reaction & $\mathrm{a}$ & b & $\begin{array}{l}\text { Adjusted } \\
\mathrm{R}^{2} \\
\end{array}$ & $\begin{array}{l}\text { TUV } \\
(\mathrm{s}-1)\end{array}$ & $\begin{array}{l}\mathrm{C}-130 \\
(\mathrm{~s}-1)\end{array}$ \\
\hline R_01 & $\mathrm{O} 3->\mathrm{O} 2+\mathrm{O}(1 \mathrm{D})$ & $2.80 \mathrm{E}-4$ & -1.75 & 0.996 & 4.47E-05 & 3.74E-05 \\
\hline R_02 & $\mathrm{O} 3->\mathrm{O} 2+\mathrm{O}(3 \mathrm{P})$ & $6.76 \mathrm{E}-4$ & -0.30 & 0.996 & 4.92E-04 & NA \\
\hline R_03 & $\mathrm{H} 2 \mathrm{O} 2$-> $2 \mathrm{OH}$ & $2.10 \mathrm{E}-5$ & -0.86 & 0.993 & 8.42E-06 & 7.96E-06 \\
\hline R_04 & $\mathrm{NO} 2->\mathrm{NO}+\mathrm{O}(3 \mathrm{P})$ & $1.88 \mathrm{E}-2$ & -0.50 & 0.999 & $1.11 \mathrm{E}-02$ & $9.48 \mathrm{E}-03$ \\
\hline R_05 & $\mathrm{NO} 3->\mathrm{NO}+\mathrm{O} 2$ & 3.03E-2 & -0.22 & 0.998 & $2.40 \mathrm{E}-02$ & NA \\
\hline R_06 & $\mathrm{NO} 3->\mathrm{NO} 2+\mathrm{O}(3 \mathrm{P})$ & $2.49 \mathrm{E}-1$ & -0.25 & 0.999 & $1.91 \mathrm{E}-01$ & NA \\
\hline R_07 & $\mathrm{N} 2 \mathrm{O} 5$-> NO3 + NO + O(3P) & $1.02 \mathrm{E}-6$ & -5.50 & 0.998 & 3.03E-09 & NA \\
\hline R_08 & N2O5 -> NO3 + NO2 & $1.25 \mathrm{E}-4$ & -0.85 & 0.994 & 5.09E-05 & 4.73E-05 \\
\hline R_09 & $\mathrm{HNO} 2->\mathrm{OH}+\mathrm{NO}$ & 3.85E-3 & -0.50 & 0.995 & $2.26 \mathrm{E}-03$ & $1.54 \mathrm{E}-03$ \\
\hline R_10 & $\mathrm{HNO} 3$-> OH + NO2 & $2.80 \mathrm{E}-6$ & -1.20 & 0.993 & 7.93E-07 & $6.82 \mathrm{E}-07$ \\
\hline R_11 & HNO4 -> HO2 + NO2 & $2.05 \mathrm{E}-5$ & -1.20 & 0.995 & $5.80 \mathrm{E}-06$ & 7.22E-06 \\
\hline R_12 & $\mathrm{CH} 2 \mathrm{O}->\mathrm{H}+\mathrm{HCO}$ & $1.05 \mathrm{E}-4$ & -1.00 & 0.996 & 3.68E-05 & 3.53E-05 \\
\hline R_13 & $\mathrm{CH} 2 \mathrm{O}->\mathrm{H} 2+\mathrm{CO}$ & $1.25 \mathrm{E}-4$ & -0.80 & 0.990 & 5.37E-05 & 5.38E-05 \\
\hline R_14 & CH3CHO -> CH3 + $\mathrm{HCO}$ & $2.95 \mathrm{E}-5$ & -1.40 & 0.996 & 6.74E-06 & 5.79E-06 \\
\hline R_15 & $\mathrm{CH} 3 \mathrm{CHO}->\mathrm{CH} 4+\mathrm{CO}$ & $1.25 \mathrm{E}-8$ & -6.00 & 0.992 & $2.16 \mathrm{E}-11$ & NA \\
\hline R_16 & $\mathrm{C} 2 \mathrm{H} 5 \mathrm{CHO}->\mathrm{C} 2 \mathrm{H} 5+\mathrm{HCO}$ & 7.35E-5 & -1.10 & 0.988 & $2.28 \mathrm{E}-05$ & $1.94 \mathrm{E}-05$ \\
\hline R_17 & $\mathrm{CH} 2(\mathrm{OH}) \mathrm{CHO}$-> Products & $2.20 \mathrm{E}-5$ & -1.20 & 0.995 & $6.23 E-06$ & NA \\
\hline R_18 & $\mathrm{CH} 2=\mathrm{C}(\mathrm{CH} 3) \mathrm{CHO}->$ Products & $1.17 \mathrm{E}-5$ & -0.60 & 0.996 & $6.21 \mathrm{E}-06$ & NA \\
\hline R_19 & СН3СОСН3 -> CH3CO + CH3 & $3.76 \mathrm{E}-6$ & -1.80 & 0.992 & 5.61E-07 & $1.20 \mathrm{E}-06$ \\
\hline R_20 & СH3COCHCH2 -> Products & $1.12 \mathrm{E}-5$ & -0.77 & 0.991 & 4.92E-06 & NA \\
\hline R_21 & $\begin{array}{l}\mathrm{CH} 3 \mathrm{COCH} 2 \mathrm{CH} 3 \text {-> CH3CO + } \\
\text { CH2CH3 }\end{array}$ & $3.05 \mathrm{E}-5$ & -1.20 & 0.994 & 8.52E-06 & 7.26E-06 \\
\hline R_22 & $\begin{array}{l}\mathrm{CH} 2(\mathrm{OH}) \mathrm{COCH} 3->\mathrm{CH} 3 \mathrm{CO}+ \\
\mathrm{CH} 2(\mathrm{OH})\end{array}$ & 4.05E-6 & -1.10 & 0.990 & $1.26 \mathrm{E}-06$ & NA \\
\hline R_23 & $\mathrm{CH} 3 \mathrm{OOH}->\mathrm{CH} 3 \mathrm{O}+\mathrm{OH}$ & $1.65 \mathrm{E}-5$ & -0.88 & 0.991 & $6.44 \mathrm{E}-06$ & NA \\
\hline R_24 & СH3CO(OOH) -> Products & $2.74 \mathrm{E}-6$ & -1.00 & 0.994 & $9.68 \mathrm{E}-07$ & NA \\
\hline R_25 & CH3ONO2 -> CH3O + NO2 & $3.94 \mathrm{E}-6$ & -1.20 & 0.994 & $1.12 \mathrm{E}-06$ & NA \\
\hline R_26 & $\begin{array}{l}\mathrm{CH} 3 \mathrm{CO}(\mathrm{OONO} 2)->\mathrm{CH} 3 \mathrm{CO}(\mathrm{OO}) \\
+\mathrm{NO} 2\end{array}$ & 6 & -1.00 & 0.992 & $5.26 \mathrm{E}-07$ & 5.54E-07 \\
\hline R_27 & CH3CO(OONO2) -> CH3CO(O) + & $1.00 \mathrm{E}-6$ & -1.00 & 0.993 & 3.51E-07 & 2.37E-07 \\
\hline
\end{tabular}




\begin{tabular}{|c|c|c|c|c|c|c|}
\hline & NO3 & & & & & \\
\hline R_28 & $\begin{array}{l}\mathrm{CH} 3 \mathrm{CH} 2 \mathrm{CO}(\mathrm{OONO} 2)-> \\
\mathrm{CH} 3 \mathrm{CH} 2 \mathrm{CO}(\mathrm{OO})+\mathrm{NO} 2\end{array}$ & $2.50 \mathrm{E}-6$ & -1.00 & 0.992 & 8.68E-07 & NA \\
\hline R_29 & CHOCHO -> HCO + HCO & $1.16 \mathrm{E}-4$ & -0.45 & 0.998 & 7.22E-05 & 7.59E-05 \\
\hline R_30 & CH3COCHO $->$ CH3CO $+\mathrm{HCO}$ & $2.60 \mathrm{E}-4$ & -0.60 & 0.996 & $1.38 \mathrm{E}-04$ & $1.45 \mathrm{E}-04$ \\
\hline
\end{tabular}

1

2

3

4

5

6

7

8

9

10

11

12

13

14

15

16

17

18

19

20

21

22

23 


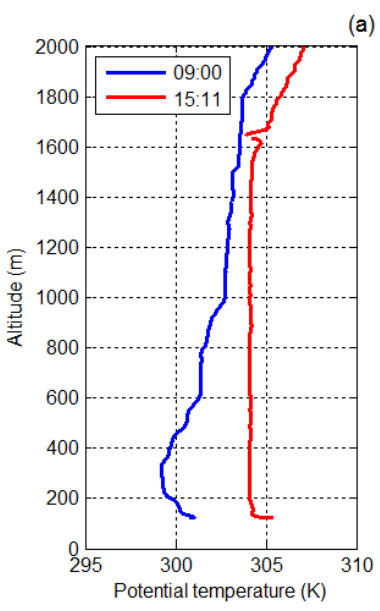

(a) 2013-6-4

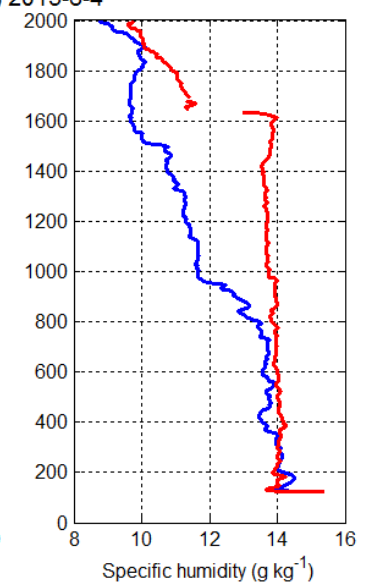

(c) 2013-6-12
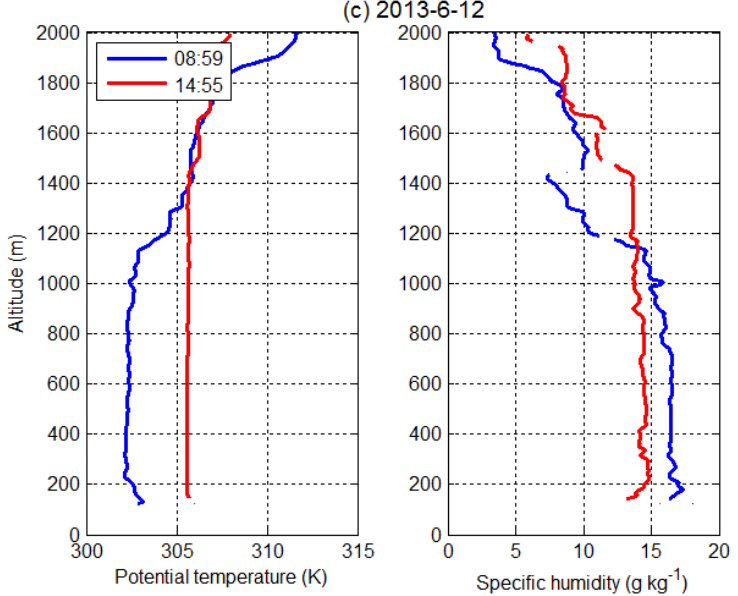

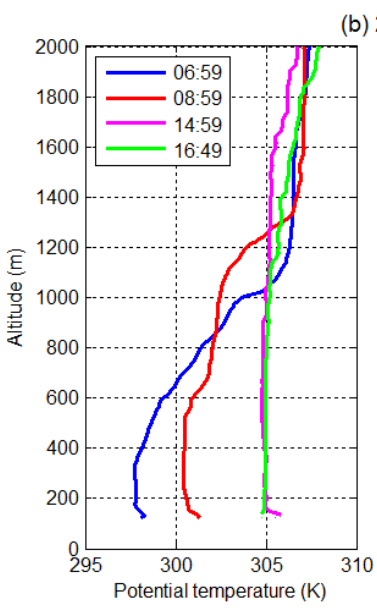

(b) $2013-6-11$
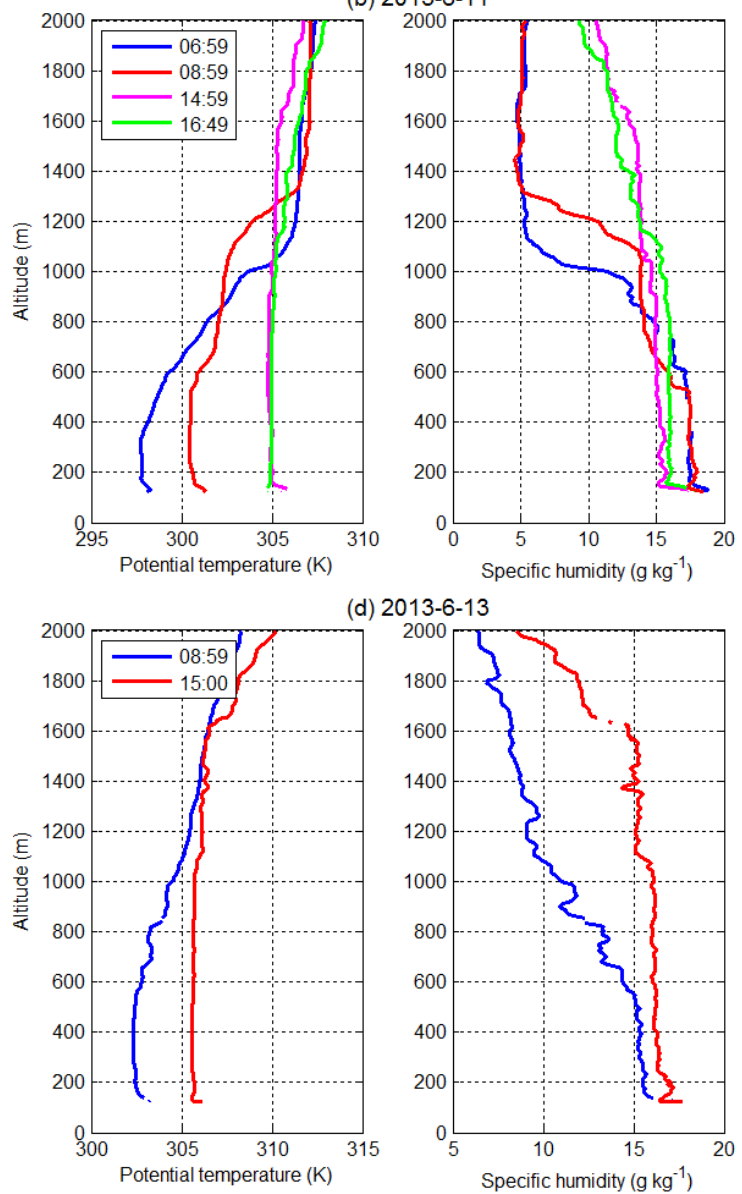

(d) $2013-6-13$

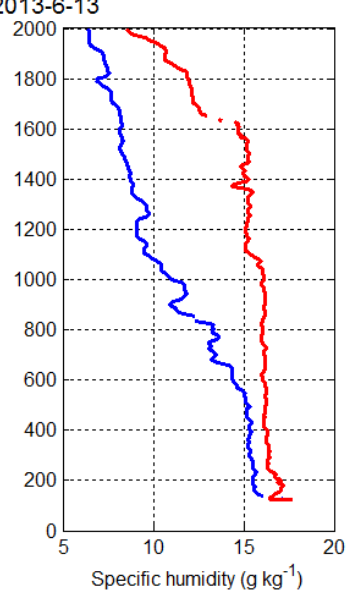

6

Figure S1. Vertical profiles of potential temperature and specific humidity collected from the sounding system at the SEARCH site on (a) 2013-6-4, (b) 2013-6-11, (c) 2013-6-12, and (d) 2013-6-13. Time series shown in the legend are in CST. Data shown here are representative of the other sampling days in this study. 

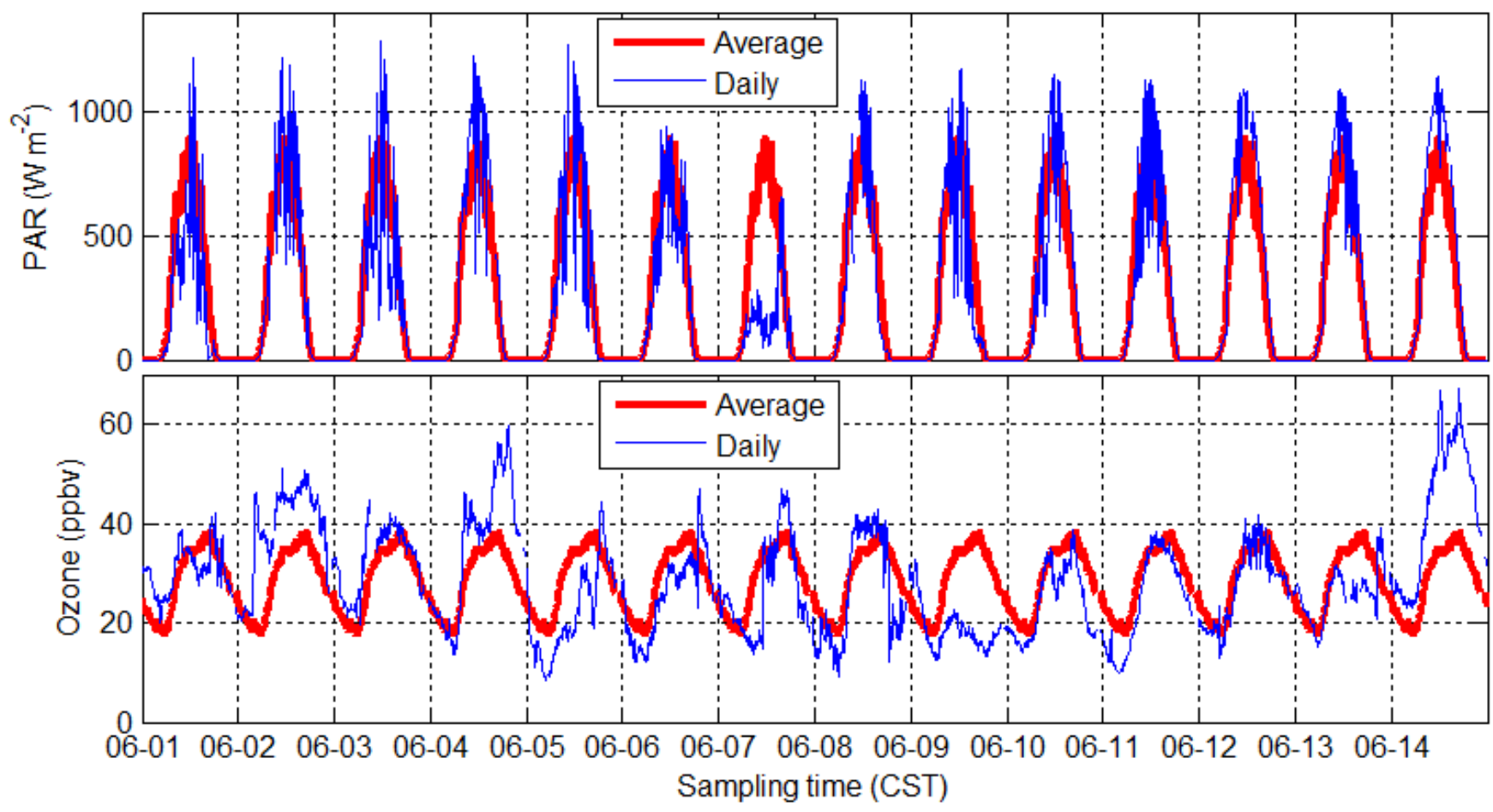

8 Figure S2. Top panel, photosynthetically active radiation (PAR) measured at $32 \mathrm{~m}$ on the 9 AABC flux tower. Bottom panel, ozone mixing ratios measured at the SEARCH site. x-axis show sampling time in month-day. Thin blue line indicates daily measurements. Bold red line indicates a one-day diurnal profile obtained by averaging the data between 2013-6-1 and 2013-6-14, which is then duplicated for each day to show the deviation of daily profile from the averaged profile. 

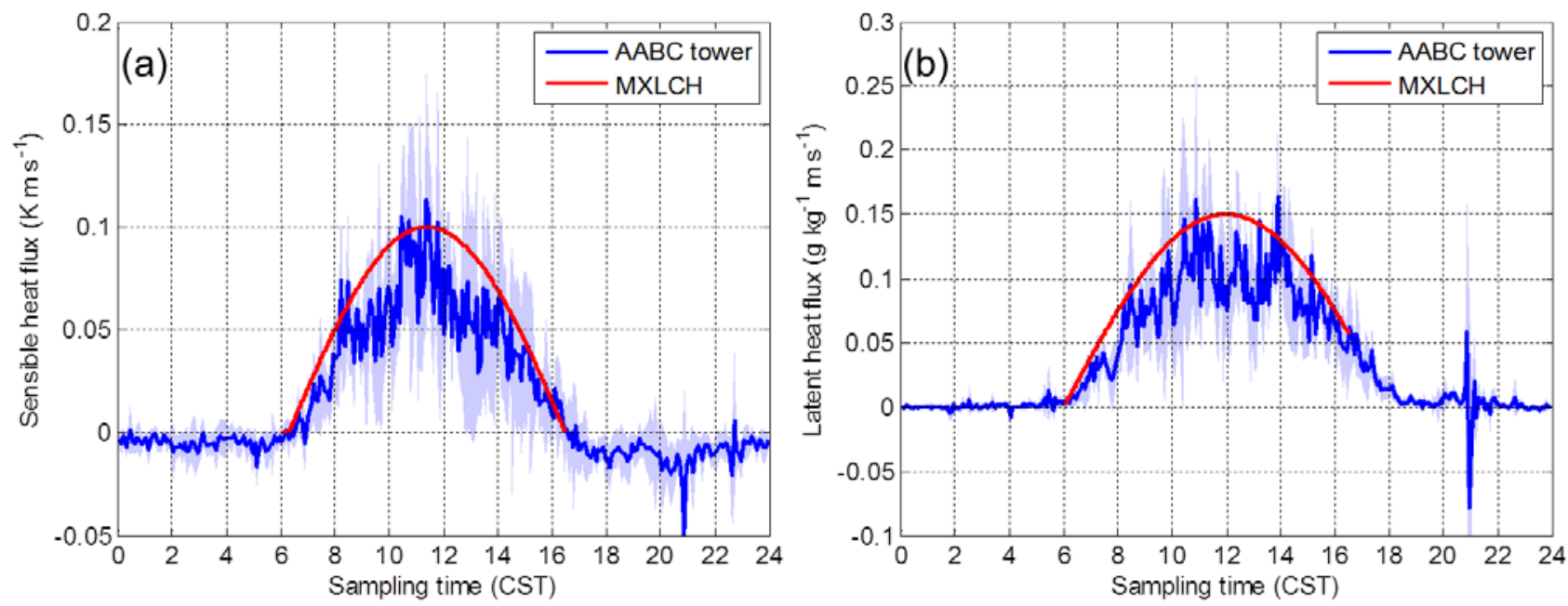

Figure S3. Diurnal evolution of (a) surface sensible heat flux, (b) surface latent heat flux. The blue lines indicate the averaged data obtained from the AABC tower over the low cloud cover days (June 10-13). The shaded areas indicate 1 standard deviation of the observations. The red line indicates the fitted curve, which is prescribed to MXLCH. 


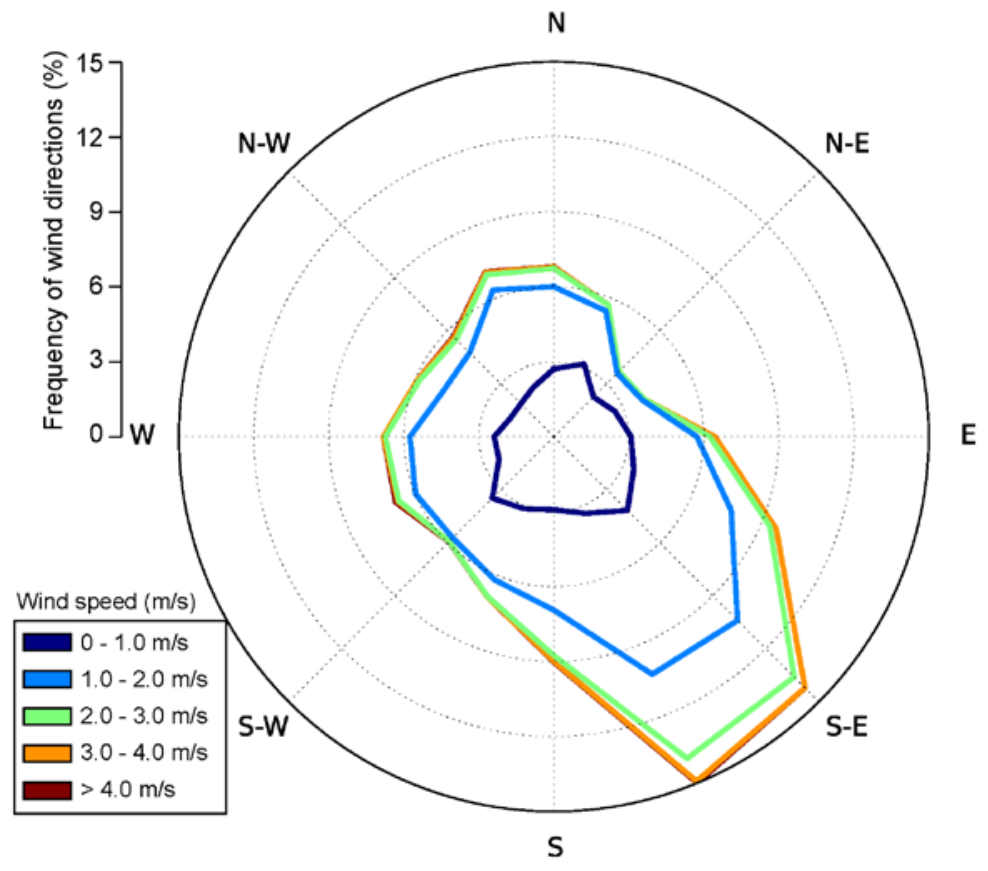

8

9 Figure S4. Summary of the horizontal wind data during 06:00-16:30 CST of the selected days 10 (June 5, 6, 8, 10-13, 2013). The wind data were collected on top of the AABC flux tower at $1144 \mathrm{~m}$ height above the ground level.

12 

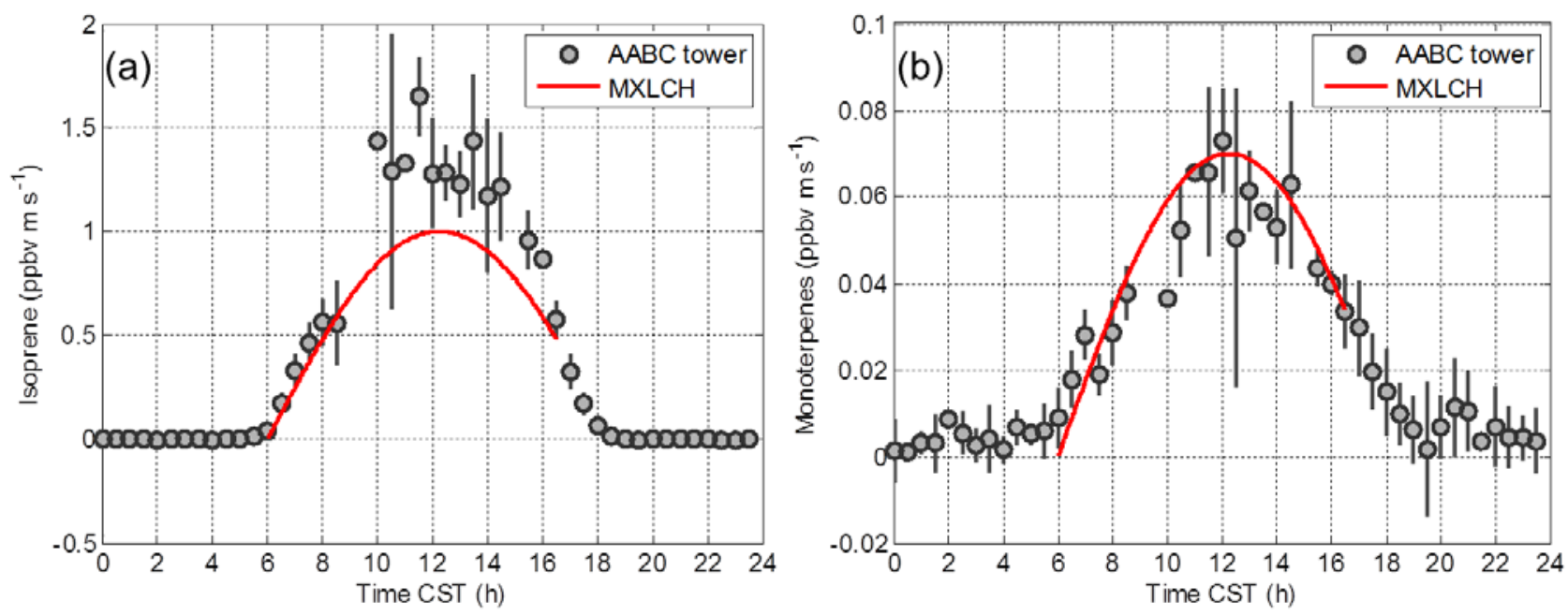

10

Figure S5. Diurnal evolution of (a) isoprene flux, (b) monoterpenes fluxes. The circles indicate averaged flux during within the time bin (30 min). Error bars indicate 1 standard deviation. The red line indicates the fitted curve, which is prescribed to MXLCH. The isoprene peak flux value prescribed in MXLCH is on the lower bound of the observations. 


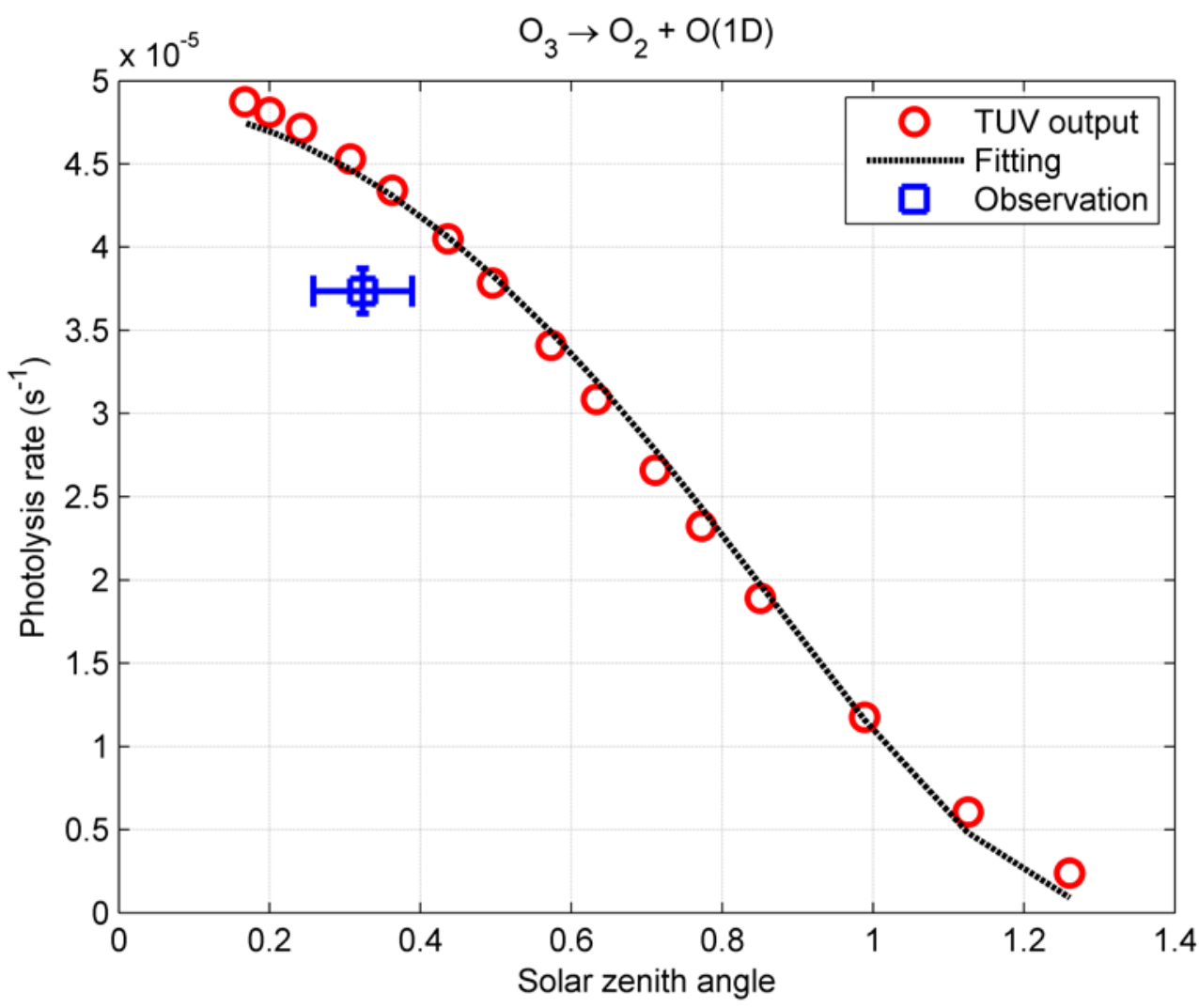

9

10 Figure S6. Comparison of TUV outputs with observation for photolysis reaction O3 -> O2 + 11 O(1D). Error bars indicate 1 standard deviation. See Table S4 for curve fitting parameters and 12 methods of processing the observational data.

13

14 

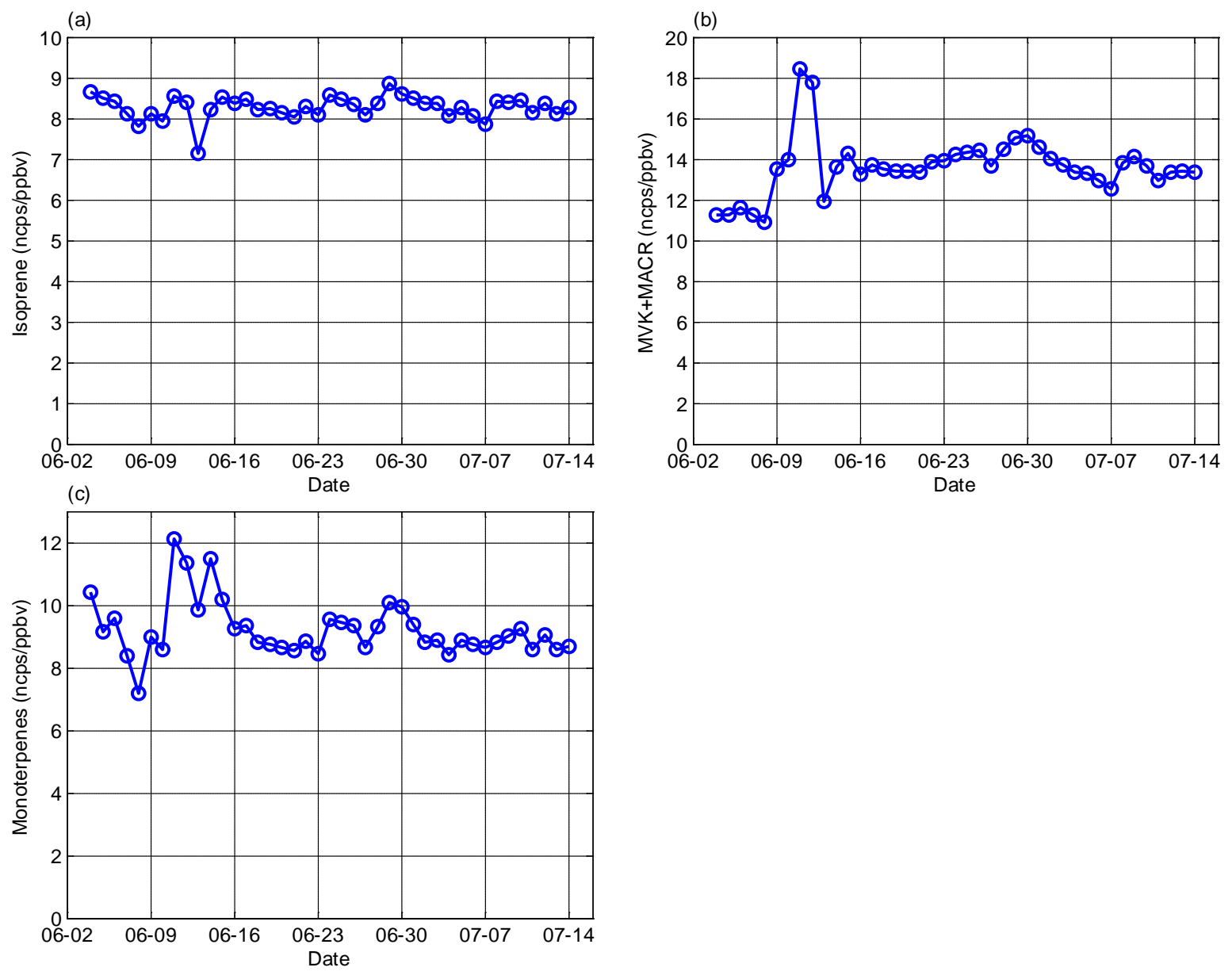

6

7 Figure S7. Summary of the sensitivities for (a) isoprene, (b) MVK+MACR, and (c) 8 monoterpenes obtained from standard gas calibrations during the whole campaign period.

9 
2 References

3 Cappellin, L., Karl, T., Probst, M., Ismailova, O., Winkler, P. M., Soukoulis, C., Aprea, E., 4 Mark, T. D., Gasperi, F., and Biasioli, F.: On Quantitative Determination of Volatile Organic 5 Compound Concentrations Using Proton Transfer Reaction Time-of-Flight Mass 6 Spectrometry, Environ Sci Technol, 46, 2283-2290, 2012.

7 Li, J. W.: Development and evaluation of flexible empirical peak functions for processing 8 chromatographic peaks, Anal Chem, 69, 4452-4462, Doi 10.1021/Ac970481d, 1997.

9 Nguyen, T. B., Crounse, J. D., Schwantes, R. H., Teng, A. P., Bates, K. H., Zhang, X., St 10 Clair, J. M., Brune, W. H., Tyndall, G. S., Keutsch, F. N., Seinfeld, J. H., and Wennberg, P. 11 O.: Overview of the Focused Isoprene eXperiment at the California Institute of Technology 12 (FIXCIT): mechanistic chamber studies on the oxidation of biogenic compounds, Atmos Chem Phys, 14, 13531-13549, 10.5194/acp-14-13531-2014, 2014. 